\title{
Navier-Stokes Equations for Compressible Fluids: Global Existence and Qualitative Properties of the Solutions in the General Case
}

\author{
Alberto Valli ${ }^{1, \star}$ and Wojciech M. Zajaczkowski ${ }^{2, \star}$ \\ 1 Dipartimento di Matematica, Università di Trento, I-38050 Povo (Trento), Italy \\ 2 Institute of Fundamental Technological Research, Polish Academy of Sciences, \\ Swietokrzyska 21, PL-00-049 Warsaw, Poland
}

\begin{abstract}
We consider the equations which describe the motion of a viscous compressible fluid, taking into consideration the case of inflow and/or outflow through the boundary. By means of some a priori estimates we prove the existence of a global (in time) solution. Moreover, as a consequence of a stability result, we show that there exist a periodic solution and a stationary solution.
\end{abstract}

\section{Introduction}

In this paper the motion of a viscous compressible fluid is considered. The motion in a bounded domain $\Omega \subset \mathbb{R}^{3}$ is described by the following equations

$$
\begin{gathered}
\varrho\left[u_{t}+u \cdot \nabla u-b\right]=-\nabla p-A u \quad \text { in } Q_{T}, \\
\varrho_{t}+u \cdot \nabla \varrho+\varrho \operatorname{div} u=0 \quad \text { in } Q_{T}, \\
\varrho c_{v}\left[\theta_{t}+u \cdot \nabla \theta\right]+\theta p_{\theta} \operatorname{div} u \\
=\varrho r+\chi \Delta \theta+\frac{\mu}{2} \sum_{i, j}\left(D_{i} u^{j}+D_{j} u^{i}\right)^{2}+(\nu-\mu)(\operatorname{div} u)^{2} \text { in } Q_{T}, \\
u_{\mid t=0}=u_{0} \text { in } \Omega, \\
u_{\mid \partial \Omega}=\bar{u}_{\mid \partial \Omega} \text { on } \Sigma_{T}, \\
\theta_{\mid t=0}=\theta_{0} \text { in } \Omega, \\
\theta_{\mid \partial \Omega}=\bar{\theta}_{\mid \partial \Omega} \text { on } \Sigma_{T}, \\
\varrho_{\mid t=0}=\varrho_{0} \text { in } \Omega,
\end{gathered}
$$

where $-A \equiv \mu \Delta+\nu \nabla \operatorname{div}$. (See, for instance, Serrin [23].)

* $\quad$ Partially supported by G.N.A.F.A of C.N.R. (Italy) 
The following notations are introduced: $\left.Q_{T} \equiv\right] 0, T\left[\times \Omega, \Sigma_{T} \equiv\right] 0, T[\times \partial \Omega$, $0<T \leqq \infty, \varrho=\varrho(t, x)$ is the density of the fluid, $u=u(t, x)$ the velocity, $b=b(t, x)$ the external force field per unit mass, $\theta=\theta(t, x)$ the absolute temperature, $r=r(t, x)$ the heat sources per unit time per unit mass, $p=p(\varrho, \theta)$ the pressure and $c_{v}=c_{v}(\varrho, \theta)$ the specific heat at constant volume. These last two are assumed to be known functions of $\varrho$ and $\theta$. The viscosity coefficients $\mu$ and $v$ and the coefficient of heat conductivity $\chi$ are assumed to be constant and to satisfy the following thermodynamic restrictions

$$
\mu>0, \quad v \geqq \frac{1}{3} \mu, \quad \chi>0 .
$$

(Actually, it is enough to assume that these coefficients are $C^{2}$ functions of $\varrho$ and $\theta$, see Appendix in Sect. 6.)

The functions $u_{0}, \varrho_{0}$, and $\theta_{0}$ are the initial data of $u, \varrho$, and $\theta$, respectively. The functions $\bar{u}_{\mid \partial \Omega}$ and $\bar{\theta}_{\mid \partial \Omega}$ are the boundary data for $u$ and $\theta$, respectively. For the sake of simplicity, we assume that $\bar{u}$ and $\bar{\theta}$ are defined in $\bar{Q}_{T}$, without considering the problem of describing precisely their space of traces.

Problem (1.1) is well posed if

$$
\bar{u} \cdot \bar{n}_{\mid \partial \Omega} \geqq 0,
$$

where $\bar{n}$ is the unit outward normal vector to the boundary. If this condition is not satisfied in some region, one needs to prescribe the value of $\varrho$ on this part of $\partial \Omega$. To clarify the situation, define

$$
S_{1}(t) \equiv\{x \in \partial \Omega \mid \bar{u}(t, x) \cdot \bar{n}(x)<0\}
$$

and

$$
S_{2}(t) \equiv\{x \in \partial \Omega \mid \bar{u}(t, x) \cdot \bar{n}(x)>0\} .
$$

(However, for reasons which will be clear later, we assume that $S_{1}$ does not depend on $t$. We remark moreover that the domain $\Omega$ does not depend on $t$.) If $S_{1} \neq \emptyset$, then the density $\varrho$ on $S_{1}$ has to be prescribed

$$
\left.\varrho_{\mid S_{1}}=\varrho_{1} \quad \text { on } \quad\right] 0, T\left[\times S_{1} .\right.
$$

The initial and boundary data for density and temperature are assumed to be positive: $\varrho_{0}=\varrho_{0}(x)>0, \theta_{0}=\theta_{0}(x)>0, \varrho_{1}=\varrho_{1}(t, x)>0$ on $\mathbb{R}^{+} \times S_{1}, \bar{\theta}=\bar{\theta}(t, x)$ $\geqq \theta_{1}>0$ on $\Sigma_{\infty}$.

Finally, the following compatibility conditions have to be assumed

$$
\begin{gathered}
\bar{u}(0, \cdot)_{\mid \partial \Omega}=u_{0 \mid \partial \Omega}, \\
\bar{\theta}(0, \cdot)_{\mid \partial \Omega}=\theta_{0 \mid \partial \Omega}, \\
\varrho_{1}(0, \cdot)=\varrho_{0 \mid S_{1}}, \\
\varrho_{1, t}(0, \cdot)=-\operatorname{div}\left(\varrho_{0} u_{0}\right)_{\mid S_{1}},
\end{gathered}
$$

and moreover,

$$
\begin{aligned}
& \dot{v}_{0 \mid \partial \Omega}=0, \\
& \dot{\omega}_{0 \mid \partial \Omega}=0
\end{aligned}
$$

[see (2.21) and (2.22) for the definition of $\dot{v}_{0}$ and $\dot{\omega}_{0}$ ]. 
In the last thirty years this problem has been intensively studied, firstly investigating uniqueness (see Graffi [8], Serrin [26], Valli [29]) and later (local in time) existence (see Nash [20], Itaya [9], Vol'pert and Hudjaev [32] for the Cauchy problem in $\mathbb{R}^{3}$; Solonnikov [27], Tani [28], Valli [30] for the CauchyDirichlet problem in a general domain with vanishing velocity on the boundary; Fiszdon and Zajaczkowski $[4,5]$ for the Cauchy-Dirichlet problem in a bounded domain too but in the case of an inflow only, i.e., $S_{1}=\partial \Omega$; Lukaszewicz $[12,13]$ for the Cauchy-Dirichlet problem in a bounded domain in the case of inflow and outflow, i.e. $\partial \Omega=S_{1} \cup S_{2}$, or $S_{1}=\emptyset$ ).

Recently, a global existence result has been proved by Matsumura and Nishida in $\mathbb{R}^{3}[15,16]$. Afterwords, Matsumura and Nishida [17, 18], Valli [31] have shown global existence in a bounded domain $\Omega$ with $u_{\mid \partial \Omega}=0$, and Fiszdon and Zajaczkowski $[4,6]$ in the case of an inflow only. (However, in $[4,6,31]$ only the case of a barotropic fluid (i.e., $p=p(\varrho)$ ) is considered.)

The case of the half-space or of an exterior domain has been studied by Matsumura and Nishida [19]. These global existence results have been obtained under the assumptions that the data of problems are small enough. The most complete results about qualitative properties of the solution have been proved in [31], where it is shown, by means of a stability argument, that there exist periodic and stationary solutions (see also Marcati and Valli [14] for the existence of almost-periodic solutions; moreover, some partial results about stationary solutions were proved by Matsumura and Nishida [17-19] when the external force field $b$ is a gradient, and by Padula [21] when the ratio $v / \mu$ is large enough).

In this paper we study global existence, stability, existence of periodic and stationary solutions for the general Eqs. (1.1), (1.5), extending the results of [31] to the non-barotropic case. Since some non-linear terms contained in $(1.1)_{3}$ are quadratic in $D u$, one needs a priori estimates more precise than those proved in [31] in such a way that the behaviour of these nonlinear terms can be controlled (see in particular Lemma 2.4). Moreover, we consider also the case in which there is an inflow and/or an outflow. These cases require some new calculations also for the local existence theorem; furthermore, we shall see later in this introduction that in these situations some qualitative results cannot be proved.

This paper is organized in the following way. In Sect. 2 we prove the local existence of a solution for the general Cauchy-Dirichlet boundary value problem (by assuming that the region $S_{1}$, where $\bar{u} \cdot \bar{n}<0$, is a closed surface; we need this condition since to obtain a priori estimates for the density $\varrho$ one has to consider some integrals on $S_{1}$ in which $\bar{u} \cdot \bar{n}$ appears at the denominator, see Sect. 2.B and in particular (2.28)). We need a local existence theorem precisely in the form of Theorem 2.5, so that we can use the a priori estimates of Sect. 3 to extend the solution up to infinity. In Sect. 2 we remark also that the uniqueness holds in a class of functions containing the global solution to be constructed later in Sect. 3 .

In Sect. 3 we prove the a priori estimates global in time, and consequently the existence of a global solution. We need, however, that there is no inflow (i.e., $S_{1}=\emptyset$ ). Otherwise, our methods would give a solution satisfying

$$
\frac{1}{2} \varrho \leqq \varrho(t, x) \leqq \frac{3}{2} \bar{\varrho} \quad \forall(t, x) \in \bar{Q}_{\infty},
$$


where

$$
\bar{\varrho} \equiv \frac{1}{|\Omega|} \int_{\Omega} \varrho_{0}(x)>0 \quad(|\Omega| \equiv \operatorname{vol}(\Omega)) .
$$

From the equation of conservation of mass one knows that

$$
\int_{\Omega} \varrho=\varrho \varrho|\Omega|-\int_{0}^{t} \int_{\partial \Omega} \bar{u} \cdot \bar{n} \varrho
$$

hence, if the total outflow $\int_{0}^{\infty}\left[\int_{S_{2}} \bar{u} \cdot \bar{n} \varrho\right]$ is finite, as we shall assume, then the integral

$$
\int_{0}^{\infty} \int_{S_{1}} \bar{u} \cdot \bar{n}
$$

is finite. But our assumption $\bar{u} \cdot \bar{n}<0$ on the closed surface $S_{1}$ gives

$$
-\bar{u}(t, x) \cdot \bar{n}(x) \geqq a_{0}(t)>0 \quad \text { on } \quad S_{1},
$$

hence $a_{0}$ would be in $L^{1}\left(\mathbb{R}^{+}\right)$. Moreover, in proving the existence of a global solution we require the smallness of $\bar{u}$, hence of $a_{0}$; but to obtain (2.28) we use that $1 / a_{0}(t)$ is bounded.

In Sect. 4 we show the asymptotic equivalence of two solutions starting from two initial data satisfying

$$
\int_{\Omega} \varrho_{0}^{(1)}=\int_{\Omega} \varrho_{0}^{(2)}
$$

In this case we need that there is no outflow, since some technical difficulties arise if the total amounts of mass of the two solutions are different.

In Sect. 5 we prove the existence of a periodic and a stationary solution in the case of the vanishing normal component of the velocity on $\partial \Omega$. An essential tool for constructing these solutions is the use of the "stability" result proved in Sect. 4.

Now we want to make precise some necessary conditions for proving the existence of periodic and stationary solutions. Suppose that $\bar{u} \cdot \bar{n} \leqq 0$ on $\partial \Omega, \bar{u} \cdot \bar{n}<0$ on $S_{1} \neq \emptyset$, and that we get a $T$-periodic solution satisfying (1.12). Then from (1.14) one gets

$$
+\infty>-\int_{0}^{\infty} \int_{\partial \Omega} \bar{u} \cdot \bar{n}=-\sum_{k=1}^{\infty} \int_{(k-1) T}^{k T} \int_{\partial \Omega} \bar{u} \cdot \bar{n}=-\sum_{k=1}^{\infty} \int_{0}^{T} \int_{\partial \Omega} \bar{u} \cdot \bar{n},
$$

which is obviously a contradiction. Hence there is no periodic solution satisfying (1.12) in the case of an inflow without an outflow. The same happens if $\bar{u} \cdot \bar{n} \geqq 0$ on $\partial \Omega, \bar{u} \cdot \bar{n}>0$ on $S_{2}(t) \neq \emptyset$ for some $t$.

The existence of a periodic solution in the presence of an inflow and an outflow at the same time is an open problem. Similar considerations hold for the stationary case, too. In this situation, if only an inflow is present, then one gets the contradiction

$$
0>\int_{S_{1}} \bar{u} \cdot \bar{n} \varrho=\int_{\partial \Omega} \bar{u} \cdot \bar{n} \varrho=0,
$$

and analogously in the case of an outflow. 
Finally, we introduce some notations. We set

$$
\begin{aligned}
m \equiv \min _{\bar{\Omega}} \varrho_{0}(x), & M \equiv \max _{\bar{\Omega}} \varrho_{0}(x), \\
\theta_{3} \equiv \min _{\bar{\Omega}} \theta_{0}(x), & \theta_{4} \equiv \max _{\bar{\Omega}} \theta_{0}(x),
\end{aligned}
$$

and these constants will be used in proving some estimates in Sect.2. We shall denote the usual norm in the Sobolev space $H^{s}(\Omega)$ (or any other equivalent norm) by $\|\cdot\|_{s}$ for $s \geqq-1$; the norm in $W^{s, p}(\Omega)$ by $\|\cdot\|_{s, p}$; the norm in $L^{q}\left(0, T ; H^{s}(\Omega)\right)$ by $[\cdot]_{q ; s ; T}$ for $1 \leqq q \leqq \infty, s \geqq-1,0<T \leqq \infty$; the norm in $L^{q}\left(0, T ; L^{k}(\Omega)\right)$ by $\|\cdot \mid\|_{q ; k ; T}$, for $1 \leqq q \leqq \infty, 1 \leqq k \leqq \infty, 0<T \leqq \infty$. The norm in $L^{\infty}(0, T ; X)$ and in $C^{0}([0, T], X)$ are denoted in the same way. Moreover, it is useful to remark that for $C^{0}([0, \infty] ; X)$ we mean $C_{B}^{0}\left(\mathbb{R}^{+} ; X\right)$, the space of continuous and bounded functions from $\mathbb{R}^{+}$to $X$.

We introduce the space

$$
\Pi_{k, p}^{l}\left(Q_{T}\right) \equiv \bigcap_{i=k}^{l} W_{p}^{l-i}\left(0, T ; H^{i}(\Omega)\right)
$$

and for a function $u$ defined in $\bar{Q}_{T}$ we set the notation

$$
|u(t)|_{l, k, J} \equiv\left(\sum_{i=k}^{l}\left\|D_{t}^{l-i} u(t)\right\|_{H^{i}(J)}^{2}\right)^{1 / 2}, \quad t \in[0, T] .
$$

Usually we shall omit the dependence of $u$ on $t$ and the symbol $J$ when $J=\Omega$.

Finally, we recall that, from the classical results of Agmon et al. [1], the norms $\|\mu \Delta v+v \nabla \operatorname{div} v\|_{k}$ and $\|v\|_{k+2}, k \in \mathbb{N}$, are equivalent for $v_{\mid \partial \Omega}=0$, since $\mu \Delta+v \nabla \operatorname{div}$ is a strongly elliptic operator.

For reasons which will be clear in the sequel, we rewrite problem (1.1), (1.5) in a new form, by the change of variables

$$
\begin{aligned}
\sigma & \equiv \varrho-\bar{\varrho}, \quad(\bar{\varrho} \text { defined in }(1.13)), \\
v & \equiv u-\bar{u} \\
\omega & \equiv \theta-\bar{\theta},
\end{aligned}
$$

and obtain

$$
\begin{aligned}
& (\sigma+\bar{\varrho}) v_{t}+A v+p_{\varrho} \nabla \sigma+p_{\theta} \nabla \omega \\
& =-(\sigma+\bar{\varrho})\left[\bar{u}_{t}+(v+\bar{u}) \cdot \nabla(v+\bar{u})-b\right]-A \bar{u}-p_{\theta} \nabla \bar{\theta} \text { in } Q_{T}, \\
& \sigma_{t}+(v+\bar{u}) \cdot \nabla \sigma+(\sigma+\varrho) \operatorname{div}(v+\bar{u})=0 \text { in } Q_{T}, \\
& (\sigma+\bar{\varrho}) c_{v} \omega_{t}-\chi \Delta \omega+(\omega+\bar{\theta}) p_{\theta} \operatorname{div} v \\
& =-(\sigma+\varrho) c_{v}\left[\bar{\theta}_{t}+(v+\bar{u}) \cdot \nabla(\omega+\bar{\theta})\right]+(\sigma+\varrho) r \\
& \quad+\frac{\mu}{2} \sum_{i, j}\left[D_{i}(v+\bar{u})^{j}+D_{j}(v+\bar{u})^{i}\right]^{2}+(v-\mu)[\operatorname{div}(v+\bar{u})]^{2}+\chi \Delta \bar{\theta} \\
& -(\omega+\bar{\theta}) p_{\theta} \operatorname{div} \bar{u} \quad \text { in } Q_{T},
\end{aligned}
$$




$$
\begin{aligned}
v_{\mid \partial \Omega} & =0 \quad \text { on } \Sigma_{T}, \\
\omega_{\mid \partial \Omega} & =0 \text { on } \Sigma_{T}, \\
v_{\mid t=0} & =v_{0} \equiv u_{0}-\bar{u}(0) \text { in } \Omega, \\
\omega_{\mid t=0} & =\omega_{0} \equiv \theta_{0}-\bar{\theta}(0) \text { in } \Omega, \\
\sigma_{\mid t=0} & =\sigma_{0} \equiv \varrho_{0}-\bar{\varrho} \text { in } \Omega, \\
\sigma_{\mid S_{1}} & \left.=\sigma_{1} \equiv \varrho_{1}-\varrho \text { on }\right] 0, T\left[\times S_{1} .\right.
\end{aligned}
$$

\section{Local Existence}

A. Case Without an Inflow

The local existence of a solution can be proved by following the methods of [31, Sect. 2] (see also [2]). At first one considers the linear problem

$$
\begin{array}{rcc}
\varrho v_{t}+A v=F & \text { in } \quad Q_{T}, \\
v_{\mid \partial \Omega}=0 \quad \text { on } \quad & \Sigma_{T}, \\
v(0)=v_{0} & \text { in } \quad \Omega,
\end{array}
$$

where

$$
A \equiv-\mu \Delta-\nu \nabla \operatorname{div}
$$

and $\tilde{\varrho}, F$, and $v_{0}$ are known functions, $0<T \leqq \infty$.

In [31] it is proved that there exists a unique solution $v$ of (2.1) under suitable assumptions. Moreover, one gets:

Lemma 2.1. Let $\partial \Omega \in C^{3}, \quad \tilde{\varrho} \in L^{\infty}\left(Q_{T}\right), \quad 0<m / 2 \leqq \tilde{\varrho}(t, x) \leqq 2 M$ a.e. in $Q_{T}$, $\tilde{\varrho}(0, x) \in L^{\infty}(\Omega), 0<m \leqq \tilde{\varrho}(0, x) \leqq M$ a.e. in $\Omega, \nabla \tilde{\varrho} \in L^{2}\left(0, T ; L^{3}(\Omega)\right), \tilde{\varrho}_{t} \in L^{2}(0, T$; $\left.L^{3}(\Omega)\right), \quad F \in L^{2}\left(0, T ; H^{1}(\Omega)\right), \quad F_{t} \in L^{2}\left(0, T ; L^{2}(\Omega)\right)$ and $v_{0} \in H_{0}^{1}(\Omega), \quad[F(0)$ $\left.-A v_{0}\right] / \tilde{\varrho}(0) \in H_{0}^{1}(\Omega)$. Then the solution $v$ of $(2.1)$ is such that $v \in L^{2}\left(0, T ; H^{3}(\Omega)\right)$ $\cap C^{0}\left([0, T] ; H^{2}(\Omega)\right), v_{t} \in L^{2}\left(0, T ; H^{2}(\Omega)\right) \cap C^{0}\left([0, T] ; H^{1}(\Omega)\right), v_{t t} \in L^{2}\left(0, T ; L^{2}(\Omega)\right)$ and

$$
\begin{aligned}
& {[v]_{\infty ; 2 ; T}^{2}+[v]_{2 ; 3 ; T}^{2}+\left[v_{t}\right]_{\infty ; 1 ; T}^{2}+\left[v_{t}\right]_{2 ; 2 ; T}^{2}+\left[v_{t t}\right]_{2 ; 0 ; T}^{2}} \\
& \leqq \\
& c_{1}\left\{[F]_{2 ; 1 ; T}^{2}+[F]_{\infty ; 0 ; T}^{2}+\left(\left[F_{t}\right]_{2 ; 0 ; T}^{2}+\left\|\frac{F(0)-A v_{0}}{\tilde{\varrho}(0)}\right\|_{1}^{2}\right)\right. \\
& \cdot\left(1+\left\|\tilde{\varrho}_{t}\left|\left\|_{2 ; 3 ; T}^{2}+\right\| \nabla \tilde{\varrho} \|\right|_{2 ; 3 ; T}^{2}\right) \exp \left(c_{1}\left\|\varrho_{t}\right\| \|_{2 ; 3 ; T}^{2}\right)\right\}
\end{aligned}
$$

Here and in the sequel each constant $c, c_{i}, C_{i}$ will depend (at most) on $\Omega, \mu, \nu, \chi, \bar{\varrho}, m$, $M, \theta_{3}, \theta_{4}$, and $c_{1}^{*}, c_{2}^{*}$ (see Lemma 2.3) and not on T. Other possible dependences will be explicitly pointed out. 
Proof. By taking the derivative in $t$ of $(2.1)_{1}$, one gets for $V=v_{t}$ :

$$
\begin{gathered}
\varrho V_{t}+A V+\tilde{\varrho}_{t} V=F_{t} \text { in } Q_{T}, \\
V=0 \text { on } \Sigma_{T}, \\
V(0)=\frac{F(0)-A v_{0}}{\varrho(0)} \text { in } \Omega .
\end{gathered}
$$

Multiplying by $V_{t}+\varepsilon_{0} A V$ ( $\varepsilon_{0}$ to be chosen in a suitable way) and integrating in $\Omega$, one gets, as in the proof of Lemma 2.1 in [31]:

$$
\begin{aligned}
c_{2} & \frac{d}{d t}\|\nabla V\|_{0}^{2}+c_{3} \frac{d}{d t}\|\operatorname{div} V\|_{0}^{2}+\left\|V_{t}\right\|_{0}^{2}+\|A V\|_{0}^{2} \\
& \leqq c\left[\left\|F_{t}\right\|_{0}^{2}+\left|\int_{\Omega} \tilde{\varrho}_{t} V \cdot\left(V_{t}+\varepsilon_{0} A V\right)\right|\right] \\
& \leqq c\left[\left\|F_{t}\right\|_{0}^{2}+\|\nabla V\|_{0}\left\|\tilde{\varrho}_{t}\right\|_{L^{3}(\Omega)}\left(\left\|V_{t}\right\|_{0}+\|A V\|_{0}\right)\right] \\
& \leqq c\left[\left\|F_{t}\right\|_{0}^{2}+\|\nabla V\|_{0}^{2}\left\|\tilde{\varrho}_{t}\right\|_{L^{3}(\Omega)}^{2}\right]+\frac{1}{2}\left\|V_{t}\right\|_{0}^{2}+\frac{1}{2}\|A V\|_{0}^{2} .
\end{aligned}
$$

Hence, by Gronwall's lemma we have:

$$
[V]_{\infty ; 1 ; T}^{2} \leqq c\left(\left[F_{t}\right]_{2 ; 0 ; T}^{2}+\|V(0)\|_{1}^{2}\right) \exp \left(c\left\|\tilde{\varrho}_{t}\right\| \|_{2 ; 3 ; T}^{2}\right),
$$

i.e.

$$
[V]_{\infty ; 1 ; T}^{2} \leqq c\left(\left[F_{t}\right]_{2 ; 0 ; T}^{2}+\left\|\frac{F(0)-A v_{0}}{\varrho}\right\|_{1}^{2}\right) \exp \left(c\|\| \tilde{\varrho}_{t}\|\|_{2 ; 3 ; T}^{2}\right) .
$$

By integrating (2.5) one gets

$$
\begin{aligned}
& {[V]_{2 ; 2 ; T}^{2}+\left[V_{t}\right]_{2 ; 0 ; T}^{2} \leqq c\left(\left[F_{t}\right]_{2 ; 0 ; T}^{2}+\left\|\frac{F(0)-A v_{0}}{\tilde{\varrho}(0)}\right\|_{1}^{2}\right)} \\
& \cdot\left(1+\left|\left\|\tilde{\varrho}_{t}\right\|\right|_{2 ; 3 ; T}^{2}\right) \exp \left(c \mid\left\|\tilde{\varrho}_{t}\right\| \|_{2 ; 3 ; T}^{2}\right) \text {. }
\end{aligned}
$$

By recalling that

one has at once

$$
V=v_{t}=\frac{F-A v}{\varrho},
$$

$$
[A v]_{\infty ; 0 ; T}^{2} \leqq c\left([F]_{\infty ; 0 ; T}^{2}+\text { right-hand side of }(2.6)\right),
$$

$$
[A v]_{2 ; 1 ; T}^{2} \leqq c\left([F]_{2 ; 1 ; T}^{2}+[\nabla \varrho \cdot V]_{2 ; 0 ; T}^{2}+\text { right-hand side of }(2.7)\right) \text {. }
$$

On the other hand

$$
\begin{aligned}
{[\nabla \tilde{\varrho} \cdot V]_{2 ; 0 ; T}^{2} } & =\int_{0}^{T}\|\nabla \tilde{\varrho} \cdot V\|_{0}^{2} \leqq c \int_{0}^{T}\|\nabla \tilde{\varrho}\|_{L^{3}(\Omega)}^{2}\|V\|_{1}^{2} \\
& \leqq\|\nabla \varrho\|_{2 ; 3 ; T}^{2} \quad \text { (right-hand side of (2.6)). }
\end{aligned}
$$

From (2.6)-(2.10) one gets at once (2.3). 
Remark 2.2. Observe that, from the assumptions on $\tilde{\varrho}$, the condition $\left[F(0)-A v_{0}\right] / \varrho(0) \in H_{0}^{1}(\Omega)$ implies that $\left[F(0)-A v_{0}\right] \in H^{1 / 2}(\Omega)$. Since $F(0) \in H^{1 / 2}(\Omega)$ (by interpolation), and $v_{0} \in H_{0}^{1}(\Omega)$, one gets at once that these conditions imply $v_{0} \in H^{5 / 2}(\Omega)$.

Consider now the linear problem

$$
\begin{array}{r}
\tilde{c}_{v} \varrho \omega_{t}-\chi \Delta \omega=G \quad \text { in } \quad Q_{T}, \\
\omega_{\mid \partial \Omega}=0 \text { on } \Sigma_{T}, \\
\omega(0)=\omega_{0} \text { in } \Omega,
\end{array}
$$

where $\tilde{c}_{v}, \tilde{\varrho}, G$, and $\omega_{0}$ are known functions.

By proceeding exactly as before, considering $-\chi \Delta$ instead of $A$, one gets

Lemma 2.3. Let $\partial \Omega \in C^{3}, \tilde{c}_{v} \tilde{\varrho} \in L^{\infty}\left(Q_{T}\right), 0<c_{1}^{*} \frac{m}{2} \leqq\left(\tilde{c}_{v} \tilde{\varrho}\right)(t, x) \leqq 2 c_{2}^{*} M$ a.e. in $Q_{T}$, $\left(\tilde{c}_{\nu} \tilde{\varrho}\right)(0, x) \in L^{\infty}(\Omega), 0<c_{1}^{*} m \leqq\left(\tilde{c}_{v} \tilde{\varrho}\right)(0, x) \leqq c_{2}^{*} M$ a.e. in $\Omega_{T}, \nabla\left(\tilde{c}_{v} \tilde{\varrho}\right) \in L^{2}\left(0, T ; L^{3}(\Omega)\right)$, $\left(\tilde{c_{v}} \tilde{\varrho}\right)_{t} \in L^{2}\left(0, T ; L^{3}(\Omega)\right), G \in L^{2}\left(0, T ; H^{1}(\Omega)\right), G_{t} \in L^{2}\left(0, T ; L^{2}(\Omega)\right)$ and $[G(0)$ $\left.+\chi \Delta \omega_{0}\right] /\left(\tilde{c}_{v} \varrho\right)(0) \in H_{0}^{1}(\Omega), \omega_{0} \in H_{0}^{1}(\Omega)$. Then the solution $\omega$ of $(2.11)$ is such that $\omega \in L^{2}\left(0, T ; \quad H^{3}(\Omega)\right) \cap C^{0}\left([0, T] ; \quad H^{2}(\Omega)\right), \quad \omega_{t} \in L^{2}\left(0, T ; \quad H^{2}(\Omega)\right) \cap C^{0}([0, T] ;$ $\left.H^{1}(\Omega)\right), \omega_{t t} \in L^{2}\left(0, T ; L^{2}(\Omega)\right)$ and

$$
\begin{aligned}
& {[\omega]_{\infty ; 2 ; T}^{2}+[\omega]_{2 ; 3 ; T}^{2}+\left[\omega_{t}\right]_{\infty ; 1 ; T}^{2}+\left[\omega_{t}\right]_{2 ; 2 ; T}^{2}+\left[\omega_{t t}\right]_{2 ; 0 ; T}^{2} } \\
& \leqq c_{4}\left\{[G]_{2 ; 1 ; T}^{2}+[G]_{\infty ; 0 ; T}^{2}+\left(\left[G_{t}\right]_{2 ; 0 ; T}^{2}+\left\|\frac{G(0)+\chi \Delta \omega_{0}}{\left(\tilde{c}_{v} \tilde{\varrho}\right)(0)}\right\|_{1}^{2}\right)\right. \\
&\left.\cdot\left(1+\mid\left\|\nabla\left(\tilde{c}_{v} \tilde{\varrho}\right)\right\|\left\|_{2 ; 3 ; T}^{2}+\right\|\left(\tilde{c}_{v} \varrho\right)_{t} \|_{2 ; 3 ; T}^{2}\right) \exp \left(\left.c_{4}\left\|\left(\tilde{c}_{v} \tilde{\varrho}\right)_{t}\right\|\right|_{2 ; 3 ; T} ^{2}\right)\right\} .
\end{aligned}
$$

Finally, consider the linear problem

$$
\begin{aligned}
\sigma_{t}+\tilde{u} \cdot \nabla \sigma+\sigma \operatorname{div} \tilde{u}+\bar{\varrho} \operatorname{div} \tilde{u} & =0 \quad \text { in } \quad Q_{T}, \\
\sigma(0) & =\sigma_{0} \quad \text { in } \Omega,
\end{aligned}
$$

where $\tilde{u}$ and $\sigma_{0}$ are known functions. One obtains

Lemma 2.4. Let $\partial \Omega \in C^{1}, \tilde{u} \in L^{1}\left(0, T ; H^{3}(\Omega)\right), \tilde{u} \cdot \bar{n} \geqq 0$ on $\Sigma_{T}$, and $\sigma_{0} \in H^{2}(\Omega)$. Then there exists a unique solution $\sigma$ of (2.13) such that $\sigma \in C^{0}\left([0, T] ; H^{2}(\Omega)\right)$ and

$$
[\sigma]_{\infty ; 2 ; T} \leqq\left(\left\|\sigma_{0}\right\|_{2}+c_{5}[\tilde{u}]_{1 ; 3 ; T}\right) \exp \left(c_{5}[\tilde{u}]_{1 ; 3 ; T}\right) \text {. }
$$

If in addition $\tilde{u} \in C^{0}\left([0, T] ; H^{2}(\Omega)\right)$, then $\sigma_{t} \in C^{0}\left([0, T] ; H^{1}(\Omega)\right)$ and

$$
\left[\sigma_{t}\right]_{\infty ; 1 ; T} \leqq c_{6}[\tilde{u}]_{\infty ; 2 ; T}\left[\left(\left\|\sigma_{0}\right\|_{2}+c_{5}[\tilde{u}]_{1 ; 3 ; T}\right) \exp \left(c_{5}[\tilde{u}]_{1 ; 3 ; T}\right)+1\right] \text {. }
$$

Moreover, if $\tilde{u} \cdot \bar{n}=0$ on $\Sigma_{T}$ and $\int_{\Omega} \sigma_{0}=0$, then $\int_{\Omega} \sigma=0$ for each $t \in[0, T]$.

Proof. The existence of the solution follows from the method of characteristics. If we define $U(t, s, x)$ to be the solution of

$$
\begin{aligned}
& \frac{d}{d t} U(t, s, x)=\tilde{u}(t, U(t, s, x)), \quad t, s \in[0, T], x \in \bar{\Omega}, \\
& U(s, s, x)=x
\end{aligned}
$$


the solution $\sigma$ is expressed by

$$
\begin{aligned}
\sigma(t, x)= & -\bar{\varrho}+\left[\sigma_{0}(U(0, t, x))+\varrho\right] \\
& \cdot \exp \left(-\int_{0}^{t} \operatorname{div} \tilde{u}(s, U(s, t, x)) d s\right) .
\end{aligned}
$$

The regularity of $U(t, s, x)$ gives $\sigma \in C^{0}\left([0, T] ; H^{2}(\Omega)\right)$ (see [30, p. 207]). To prove estimate (2.14) one multiplies Eq. (2.13) 1 by $\sigma$ and integrates in $\Omega$. Integrating by parts the term $\int_{\Omega} \tilde{u} \cdot \nabla \sigma \sigma$, one has

$$
\frac{1}{2} \frac{d}{d t}\|\sigma\|_{0}^{2} \leqq \frac{1}{2} \int_{\Omega} \operatorname{div} \tilde{u} \sigma^{2}+\varrho \int_{\Omega}|\operatorname{div} \tilde{u}||\sigma|-\frac{1}{2} \int_{\partial \Omega} \tilde{u} \cdot \bar{n} \sigma^{2} .
$$

By the assumption $\tilde{u} \cdot \bar{n} \geqq 0$ on $\partial \Omega$, the boundary term can be omitted. One proceeds in the same way also for the first and the second derivatives, always integrating by parts the terms $\int_{\Omega}[(\tilde{u} \cdot \nabla) \nabla \sigma] \nabla \sigma$ and $\int_{\Omega} \tilde{u} \cdot \nabla\left(D_{k} D_{h} \sigma\right) D_{k} D_{h} \sigma$ (see for instance [31, Lemma 2.3]), and one gets

$$
\frac{1}{2} \frac{d}{d t}\|\sigma\|_{2}^{2} \leqq c\|\tilde{u}\|_{3}\|\sigma\|_{2}^{2}+\varrho\|\operatorname{div} \tilde{u}\|_{2}\|\sigma\|_{2} .
$$

From Gronwall's lemma one obtains

$$
\|\sigma\|_{2} \leqq\left(\left\|\sigma_{0}\right\|_{2}+c \int_{0}^{T}\|\tilde{u}\|_{3}\right) \exp \left(c \int_{0}^{T}\|\tilde{u}\|_{3}\right),
$$

that is (2.14). The proof of (2.15) is trivial.

Finally, if $\tilde{u} \cdot \bar{n}=0$ on $\Sigma_{T}$ and $\int_{\Omega} \sigma_{0}=0$, from Eq. $(2.13)_{1}$ one has at once

$$
\int_{\Omega} \sigma=\int_{\Omega} \sigma_{0}-\int_{0}^{t} \int_{\partial \Omega} \tilde{u} \cdot \bar{n}(\sigma+\bar{\varrho})=0 \quad \text { for each } \quad t \in[0, T] .
$$

We are now in a position to prove the local existence of a solution to problem (1.17) (for $S_{1}=\emptyset$ ). Take $0<T<\infty$ and define

$$
\begin{aligned}
R_{T} \equiv & \left\{(\tilde{v}, \tilde{\sigma}, \tilde{\omega}) \mid \tilde{v} \in C^{0}\left([0, T] ; H^{2}(\Omega)\right) \cap L^{2}\left(0, T ; H^{3}(\Omega)\right),\right. \\
& \tilde{v}_{t} \in L^{\infty}\left(0, T ; H^{1}(\Omega)\right) \cap L^{2}\left(0, T ; H^{2}(\Omega)\right), \\
& \tilde{\sigma} \in L^{\infty}\left(0, T ; H^{2}(\Omega)\right), \tilde{\sigma}_{t} \in L^{\infty}\left(0, T ; H^{1}(\Omega)\right), \\
& \tilde{\omega} \in C^{0}\left([0, T] ; H^{2}(\Omega)\right) \cap L^{2}\left(0, T ; H^{3}(\Omega)\right), \\
& \tilde{\omega}_{t} \in L^{\infty}\left(0, T ; H^{1}(\Omega)\right) \cap L^{2}\left(0, T ; H^{2}(\Omega)\right), \\
& {[\tilde{v}]_{\infty ; 2 ; T}^{2}+[\tilde{v}]_{2 ; 3 ; T}^{2}+\left[\tilde{v}_{t}\right]_{\infty ; 1 ; T}^{2}+\left[\tilde{v}_{t}\right]_{2 ; 2 ; T}^{2} \leqq B_{1}, } \\
& \tilde{v}(0)=u_{0}-\tilde{u}(0) \text { in } \Omega, \tilde{v}=0 \text { on } \Sigma_{T}, \\
& {[\tilde{\sigma}]_{\infty ; 2 ; T \leqq} \leqq,\left[\tilde{\sigma}_{t}\right]_{\infty ; 1 ; T \leqq} B_{2}, } \\
& \tilde{\sigma}(0)=\varrho_{0}-\tilde{\varrho}, 0<\frac{m}{2} \leqq \tilde{\sigma}(t, x)+\varrho \leqq 2 M \text { a.e. in } Q_{T}, \\
& {[\tilde{\omega}]_{\infty ; 2 ; T}^{2}+[\tilde{\omega}]_{2 ; 3 ; T}^{2}+\left[\tilde{\omega}_{t}\right]_{\infty ; 1 ; T}^{2}+\left[\tilde{\omega}_{t}\right]_{2 ; 2 ; T \leqq}^{2} \leqq B_{1}, } \\
& \left.\tilde{\omega}(0)=\theta_{0}-\bar{\theta}(0) \text { in } \Omega, \tilde{\omega}=0 \text { on } \Sigma_{T}, 0<\frac{\theta_{3}}{2} \leqq \tilde{\omega}(t, x)+\bar{\theta}(t, x) \leqq 2 \theta_{4} \text { in } \bar{Q}_{T}\right\},
\end{aligned}
$$


where $B_{1}$ and $B_{2}$ will be chosen in the sequel [see (2.17), (2.19), (2.20)]. If $B_{1}$ is large enough and $T$ is sufficiently small, it is clear that $R_{T} \neq \emptyset$. In fact, let $v^{*}$ be the solution of

$$
\begin{aligned}
v_{t}^{*}-\Delta v^{*} & =H \quad \text { in } \quad Q_{\infty}, \\
v^{*} & =0 \quad \text { on } \quad \Sigma_{\infty}, \\
v^{*}(0) & =u_{0}-\bar{u}(0) \quad \text { in } \Omega,
\end{aligned}
$$

where $H \in L^{2}\left(\mathbb{R}^{+} ; H^{1}(\Omega)\right), H_{t} \in L^{2}\left(\mathbb{R}^{+} ; L^{2}(\Omega)\right)$ and $H(0)=-\Delta\left[u_{0}-\bar{u}(0)\right]$ in $\Omega$ (so that $v_{t}^{*}(0)=0$; see Lions-Magenes [11, p. 25-27] for the construction of such a function and the estimates for the norms of $H$ and $H_{t}$ ), and let $\omega^{*}$ be the solution of

$$
\begin{aligned}
\omega_{t}^{*}-\Delta \omega^{*} & =K \text { in } Q_{\infty}, \\
\omega^{*} & =0 \text { on } \Sigma_{\infty}, \\
\omega^{*}(0) & =\theta_{0}-\bar{\theta}(0) \text { in } \Omega,
\end{aligned}
$$

where $K \in L^{2}\left(\mathbb{R}^{+} ; H^{1}(\Omega)\right), K_{t} \in L^{2}\left(\mathbb{R}^{+} ; L^{2}(\Omega)\right)$, and $K(0)=-\Delta\left[\theta_{0}-\bar{\theta}(0)\right]$ in $\Omega$ (so that $\left.\omega_{t}^{*}(0)=0\right)$. Then one has

$$
\begin{aligned}
{\left[v^{*}\right]_{\infty ; 2 ; \infty}^{2} } & +\left[v^{*}\right]_{2 ; 3 ; \infty}^{2}+\left[v_{t}^{*}\right]_{\infty ; 1 ; \infty}^{2} \\
& +\left[v_{t}^{*}\right]_{2 ; 2 ; \infty}^{2} \leqq c_{7}\left\|u_{0}-\bar{u}(0)\right\|_{5 / 2}^{2},
\end{aligned}
$$

and

$$
\begin{aligned}
{\left[\omega^{*}\right]_{\infty ; 2 ; \infty}^{2} } & +\left[\omega^{*}\right]_{2 ; 3 ; \infty}^{2}+\left[\omega_{t}^{*}\right]_{\infty ; 1 ; \infty}^{2} \\
& +\left[\omega_{t}^{*}\right]_{2 ; 2 ; \infty}^{2} \leqq c_{7}\left\|\theta_{0}-\bar{\theta}(0)\right\|_{5 / 2}^{2}
\end{aligned}
$$

By taking

$$
B_{1}>\max \left\{c_{7}\left\|u_{0}-\bar{u}(0)\right\|_{5 / 2}^{2} ; c_{7}\left\|\theta_{0}-\bar{\theta}(0)\right\|_{5 / 2}^{2} ;\left\|\varrho_{0}-\bar{\varrho}\right\|_{2}\right\},
$$

one sees that $\left(v^{*}, \varrho_{0}-\bar{\varrho}, \omega^{*}\right)$ satisfy all the conditions stated in the definition of $R_{T}$, with the exception of

$$
0<\frac{\theta_{3}}{2} \leqq \omega^{*}(t, x)+\bar{\theta}(t, x) \leqq 2 \theta_{4} \quad \text { in } \quad \bar{Q}_{T} .
$$

However, we have, by interpolation,

$$
\begin{aligned}
\left\|\omega^{*}+\bar{\theta}-\theta_{0}\right\|_{C^{0}\left(\bar{Q}_{T}\right) \leqq} & c\left[\omega^{*}+\bar{\theta}-\theta_{0}\right]_{\infty ; 1 ; T}^{1 / 3}\left[\omega^{*}+\bar{\theta}-\theta_{0}\right]_{\infty ; 2 ; T}^{2 / 3} \\
\leqq & c T^{1 / 3}\left(\left[\omega_{t}^{*}\right]_{\infty ; 1 ; T}+\left[\bar{\theta}_{t}\right]_{\infty ; 1 ; T}\right)^{1 / 3} \\
& \cdot\left(\left[\omega^{*}\right]_{\infty ; 2 ; T}+[\bar{\theta}]_{\infty ; 2 ; T}+\left\|\theta_{0}\right\|_{2}\right)^{2 / 3}
\end{aligned}
$$

Hence by choosing $T$ small enough (say $T \leqq T_{1}, T_{1}$ depending on $\Omega, \theta_{3}, \theta_{4}, \bar{\theta}$, and $\left.\left\|\theta_{0}-\bar{\theta}(0)\right\|_{5 / 2}\right)$, we get (2.18). From now on we shall assume that $T \leqq T_{1}$ and that $B_{1}$ satisfies (2.17).

Consider now the map $L$ defined in $R_{T}$ in this way:

$$
L:(\tilde{v}, \tilde{\sigma}, \tilde{\omega}) \rightarrow(v, \sigma, \omega),
$$


where $v, \sigma$, and $\omega$ are the solutions of (2.1), (2.13), and (2.11), respectively, with

$$
\begin{aligned}
\tilde{\varrho} \equiv & \tilde{\sigma}+\bar{\varrho} \\
F \equiv & (\tilde{\sigma}+\bar{\varrho})\left[b-\bar{u}_{t}-(\bar{u} \cdot \nabla) \bar{u}-(\tilde{v} \cdot \nabla) \bar{u}-(\bar{u} \cdot \nabla) \tilde{v}\right. \\
& -(\tilde{v} \cdot \nabla) \tilde{v}]-A \bar{u}-\nabla[p(\tilde{\sigma}+\bar{\varrho}, \tilde{\omega}+\bar{\theta})], \\
v_{0} \equiv & u_{0}-\bar{u}(0), \\
\tilde{c}_{v} \equiv & c_{v}(\tilde{\sigma}+\bar{\varrho}, \tilde{\omega}+\bar{\theta}), \\
G \equiv & -\tilde{c}_{v}(\tilde{\sigma}+\varrho \bar{\varrho})[\bar{\theta}+\tilde{v} \cdot \nabla \tilde{\omega}+\bar{u} \cdot \nabla \tilde{\omega}+\tilde{v} \cdot \nabla \bar{\theta} \\
& +\bar{u} \cdot \nabla \bar{\theta}]-(\tilde{\omega}+\bar{\theta}) p_{\theta}(\tilde{\sigma}+\bar{\varrho}, \tilde{\omega}+\bar{\theta}) \operatorname{div}(\tilde{v}+\bar{u}) \\
& +\chi \Delta \bar{\theta}+(\tilde{\sigma}+\bar{\varrho}) r+\frac{\mu}{2} \sum_{i, k}\left(D_{k} \tilde{v}^{i}+D_{k} \bar{u}^{i}+D_{i} \tilde{v}^{k}+D_{i} \bar{u}^{k}\right)^{2} \\
& +(v-\mu)(\operatorname{div} \tilde{v}+\operatorname{div} \bar{u})^{2}, \\
\omega_{0} \equiv & \theta_{0}-\bar{\theta}(0), \quad \tilde{u} \equiv \tilde{v}+\bar{u}, \quad \sigma_{0} \equiv \varrho_{0}-\bar{\varrho} .
\end{aligned}
$$

Notice that, from this choice of $\tilde{c}_{v}$, we get

$$
c_{1}^{*} \equiv \min _{\Lambda_{1}} c_{v} \leqq \tilde{c}_{v}=c_{v}(\tilde{\sigma}+\bar{\varrho}, \tilde{\omega}+\bar{\theta}) \leqq \max _{\Lambda_{1}} c_{v} \equiv c_{2}^{*},
$$

where $\Lambda_{1} \equiv\left\{(\varrho, \theta) \in \mathbb{R}^{2} \mid \frac{m}{2} \leqq \varrho \leqq 2 M, \frac{\theta_{3}}{2} \leqq \theta \leqq 2 \theta_{4}\right\}$. Hence $c_{1}^{*}$ and $c_{2}^{*}$ depend only on the function $c_{v}$ and on $m, M, \theta_{3}, \theta_{4}$. We shall prove that $L$ has a fixed point in $R_{T}$ for $T$ small enough. We need at first some estimates, which can be obtained by means of some long but straightforward calculations:

$$
[F]_{2 ; 1 ; T}^{2} \leqq C_{1}\left(B_{1}, \bar{u}, \bar{\theta}, p\right)\left([b]_{2 ; 1 ; T}^{2}+[\bar{u}]_{2 ; 3 ; T}^{2}+T\right),
$$

$$
\begin{gathered}
{[F]_{\infty ; 0 ; T}^{2} \leqq} \\
\quad C_{2}(\bar{u}, \bar{\theta}, p) \\
\cdot\left([b]_{\infty ; 0 ; T}^{2}+1+\left\|u_{0}-\bar{u}(0)\right\|_{2}^{4}+\left\|\sigma_{0}\right\|_{1}^{2}+\left\|\theta_{0}-\bar{\theta}(0)\right\|_{1}^{2}+c\left(B_{1}, B_{2}\right) T^{2}\right), \\
{\left[F_{t}\right]_{2 ; 0 ; T}^{2} \leqq} \\
\quad C_{3}\left(B_{1}, B_{2}, \bar{u}, \bar{\theta}, p\right) \\
\cdot\left([b]_{2 ; 1 ; T}^{2}+\left[b_{t}\right]_{2 ; 0 ; T}^{2}+\left[\bar{u}_{t}\right]_{2 ; 2 ; T}^{2}+\left[\bar{u}_{t t}\right]_{2 ; 0 ; T}^{2}+T\right), \\
{[G]_{2 ; 1 ; T}^{2} \leqq C_{4}\left(B_{1}, \bar{u}, \bar{\theta}, p, c_{v}\right)\left([r]_{2 ; 1 ; T}^{2}+[\bar{\theta}]_{2 ; 3 ; T}^{2}+T+T^{1 / 2}\right),}
\end{gathered}
$$

$[G]_{\infty ; 0 ; T}^{2} \leqq C_{5}\left(\bar{u}, \bar{\theta}, p, c_{v}\right)$

$$
\cdot\left([r]_{\infty ; 0 ; T}^{2}+1+\left\|u_{0}-\bar{u}(0)\right\|_{2}^{4}+\left\|\theta_{0}-\bar{\theta}(0)\right\|_{2}^{4}+c\left(B_{1}, B_{2}\right) T\right),
$$

$\left[G_{t}\right]_{2 ; 0 ; T}^{2} \leqq C_{6}\left(B_{1}, B_{2}, \bar{u}, \bar{\theta}, p, c_{v}\right)$

$$
\cdot\left([r]_{2 ; 1 ; T}^{2}+\left[r_{t}\right]_{2 ; 0 ; T}^{2}+\left[\bar{\theta}_{t}\right]_{2 ; 2 ; T}^{2}+\left[\bar{\theta}_{t t}\right]_{2 ; 0 ; T}^{2}+T+T^{1 / 2}\right) .
$$

In the fourth and in the sixth estimate for controlling the terms which are quadratic in $D \tilde{v}$ and $D \bar{u}$ one uses

$$
\|f g\|_{0}^{2} \leqq c\|f\|_{0}\|f\|_{1}\|g\|_{1}^{2}
$$


consequently

$$
[f g]_{2 ; 0 ; T}^{2} \leqq c[g]_{\infty ; 1 ; T}^{2}[f]_{\infty ; 0 ; T}[f]_{2 ; 1 ; T} .
$$

In the second and in the fifth estimate one uses

$$
\|\tilde{v}(t)\|_{k}^{2} \leqq c\left(\|\tilde{v}(0)\|_{k}^{2}+t \int_{0}^{t}\left\|\tilde{v}_{t}(s)\right\|_{k}^{2} d s\right), \quad k=1,2 ;
$$

and the same argument for $\tilde{\sigma}$ and $\tilde{\omega}$. Finally, observe that the constants $C_{i}$ depend on $p$ and $c_{v}$ in terms of $\|p\|_{C^{2}\left(\Lambda_{1}\right)}$ and $\left\|c_{v}\right\|_{C^{1}\left(\Lambda_{1}\right)}$, and on the norms of $\bar{u}$ and $\bar{\theta}$ which are specified in the following Theorem 2.5 .

Moreover,

$$
\begin{gathered}
\|\mid \nabla \tilde{\varrho}\|\left\|_{2 ; 3 ; T}^{2}+\right\| \tilde{\varrho}_{t}\|\|_{2 ; 3 ; T}^{2} \leqq C_{7}\left(B_{1}, B_{2}\right) T, \\
\|\| \nabla\left(\tilde{c}_{v} \varrho\right) \mid\left\|_{2 ; 3 ; T}^{2}+\right\|\left(\tilde{c}_{v} \varrho\right)_{t}\|\|_{2 ; 3 ; T}^{2} \leqq C_{8}\left(B_{1}, B_{2}, \bar{\theta}, c_{v}\right) T .
\end{gathered}
$$

Finally, from (2.14) and (2.15)

$$
\begin{aligned}
& {[\sigma]_{\infty ; 2 ; T} \leqq\left(\left\|\sigma_{0}\right\|_{2}+C_{9}\left(B_{1}, \bar{u}\right) T^{1 / 2}\right) \exp \left(C_{9}\left(B_{1}, \bar{u}\right) T^{1 / 2}\right),} \\
& {\left[\sigma_{t}\right]_{\infty ; 1 ; T} \leqq c_{6}\left(\left\|\sigma_{0}\right\|_{2}+1\right) C_{10}\left(B_{1}, \bar{u}\right) \exp \left(C_{9}\left(B_{1}, \bar{u}\right) T^{1 / 2}\right) .}
\end{aligned}
$$

Hence, if we take

$$
\begin{gathered}
B_{1}>\max \left\{c _ { 1 } \left[C _ { 2 } ( \overline { u } , \overline { \theta } , p ) \left(1+\|b(0)\|_{0}^{2}+\left\|u_{0}-\bar{u}(0)\right\|_{2}^{4}+\left\|\sigma_{0}\right\|_{1}^{2}\right.\right.\right. \\
\left.\left.+\left\|\theta_{0}-\bar{\theta}(0)\right\|_{1}^{2}\right)+\left\|v_{t}(0)\right\|_{1}^{2}\right], \quad\left\|\sigma_{0}\right\|_{2}, \\
c_{4}\left[C _ { 5 } ( \overline { u } , \overline { \theta } , p , c _ { v } ) \left(1+\|r(0)\|_{0}^{2}+\left\|u_{0}-\bar{u}(0)\right\|_{2}^{4}\right.\right. \\
\left.\left.\left.+\left\|\theta_{0}-\bar{\theta}(0)\right\|_{2}^{4}\right)+\left\|\omega_{t}(0)\right\|_{1}^{2}\right]\right\}, \\
B_{2}>c_{6}\left(\left\|\sigma_{0}\right\|_{2}+1\right) C_{10}\left(B_{1}, \bar{u}\right),
\end{gathered}
$$

and $T$ small enough, we get that $v, \sigma$, and $\omega$ satisfy the estimates required in the definition of the set $R_{T}$. We want to specify that in $(2.19) v_{t}(0)$ and $\omega_{t}(0)$ are calculated from the equations and depend only on the data of the problem, i.e.,

$$
\begin{aligned}
v_{t}(0)=\frac{1}{\varrho_{0}}\{ & \left.-\nabla\left[p\left(\varrho_{0}, \theta_{0}\right)\right]-A u_{0}\right\}-\bar{u}_{t}(0)-\left(u_{0} \cdot \nabla\right) u_{0}+b(0) \equiv \dot{v}_{0}, \\
\omega_{t}(0)= & \frac{1}{c_{v}\left(\varrho_{0}, \theta_{0}\right) \varrho_{0}}\left\{-\theta_{0} p_{\theta}\left(\varrho_{0}, \theta_{0}\right) \operatorname{div} v_{0}+\chi \Delta \theta_{0}\right. \\
& \left.+\frac{\mu}{2} \sum_{i, k}\left(D_{k} u_{0}^{i}+D_{i} u_{0}^{k}\right)^{2}+(v-\mu)\left(\operatorname{div} u_{0}\right)^{2}+\varrho_{0} r(0)\right\} \\
& -\bar{\theta}_{t}(0)-u_{0} \cdot \nabla \theta_{0} \equiv \dot{\omega}_{0} .
\end{aligned}
$$

In the sequel we shall use frequently this short notation. One has also, by well known interpolation results,

$$
\begin{aligned}
&\left\|\sigma-\sigma_{0}\right\|_{C^{0}\left(\bar{Q}_{T}\right)} \leqq c\left[\sigma-\sigma_{0}\right]_{\infty ; 1 ; T}^{1 / 3}\left[\sigma-\sigma_{0}\right]_{\infty ; 2 ; T}^{2 / 3} \\
& \\
& \leqq c T^{1 / 3}\left[\sigma_{t}\right]_{\infty ; 1 ; T}^{1 / 3}\left(B_{1}+\left\|\sigma_{0}\right\|_{2}\right)^{2 / 3} \\
& \leqq c T^{1 / 3} B_{2}^{1 / 3}\left(B_{1}+\left\|\sigma_{0}\right\|_{2}\right)^{2 / 3}
\end{aligned}
$$


Hence, if $T$ is small enough,

$$
\frac{m}{2} \leqq \sigma(t, x)+\bar{\varrho} \leqq 2 M \quad \text { in } \quad \bar{Q}_{T} .
$$

Finally, repeating the argument for proving (2.18), one gets that (2.18) holds also for $\omega$.

We have shown in this way that $L\left(R_{T}\right) \subset R_{T}$ for $T$ small enough, say $T=T^{*}>0$.

Now one uses Schauder's fixed point theorem. It is clear that $R_{T^{*}}$ is convex, and it is closed in $\mathbb{X} \equiv C^{0}\left(\left[0, T^{*}\right] ; H^{1}(\Omega)\right) \times C^{0}\left(\left[0, T^{*}\right] ; H^{1}(\Omega)\right) \times C^{0}\left(\left[0, T^{*}\right] ; H^{1}(\Omega)\right)$. Moreover, from Ascoli's theorem, $R_{T^{*}}$ is compact in $\mathbb{X}$. Hence one needs only to prove that $L$ is continuous in $\mathbb{X}$. Suppose that $\left(\tilde{v}_{n}, \tilde{\sigma}_{n}, \tilde{\omega}_{n}\right) \in R_{T^{*}},\left(\tilde{v}_{n}, \tilde{\sigma}_{n}, \tilde{\omega}_{n}\right)$ $\rightarrow(\tilde{v}, \tilde{\sigma}, \tilde{\omega})$ in $\mathbb{X}$ and set $\left(v_{n}, \sigma_{n}, \omega_{n}\right) \equiv L\left(\tilde{v}_{n}, \tilde{\sigma}_{n}, \tilde{\omega}_{n}\right),(v, \sigma, \omega) \equiv L(\tilde{v}, \tilde{\sigma}, \tilde{\omega})$. Take the difference between the equations for $\left(v_{n}, \sigma_{n}, \omega_{n}\right)$ and $(v, \sigma, \omega)$, multiply by $\left(v_{n}-v\right)$, $\left(\sigma_{n}-\sigma\right)$, and $\left(\omega_{n}-\omega\right)$, respectively, and integrate in $\Omega$. By an energy argument and by Gronwall's lemma it is easily seen that $\left(v_{n}, \sigma_{n}, \omega_{n}\right)$ converge to $(v, \sigma, \omega)$ in $C^{0}\left(\left[0, T^{*}\right] ; L^{2}(\Omega)\right)$. Since $R_{T^{*}}$ is compact, $\left(v_{n}, \sigma_{n}, \omega_{n}\right)$ converge indeed to $(v, \sigma, \omega)$ in $\mathbb{X}$. Hence $L$ is continuous, and consequently has a fixed point, which is the solution of problem (1.17) in $Q_{T^{*}}\left(\right.$ for $\left.S_{1}=\emptyset\right)$.

We have thus proved the following theorem.

Theorem 2.5. Let $\partial \Omega \in C^{3}, \quad b \in L_{\mathrm{loc}}^{2}\left(\mathbb{R}^{+} ; H^{1}(\Omega)\right), \quad b_{t} \in L_{\mathrm{loc}}^{2}\left(\mathbb{R}^{+} ; L^{2}(\Omega)\right)$, $r \in L_{\mathrm{loc}}^{2}\left(\mathbb{R}^{+} ; H^{1}(\Omega)\right), r_{t} \in L_{\mathrm{loc}}^{2}\left(\mathbb{R}^{+} ; L^{2}(\Omega)\right), p=p(\varrho, \theta) \in C^{2}, c_{v}=c_{v}(\varrho, \theta) \in C^{1}, c_{v}>0$ for $\varrho>0$ and $\theta>0,\left[u_{0}-\bar{u}(0)\right] \in H_{0}^{1}(\Omega), \dot{v}_{0} \in H_{0}^{1}(\Omega)(\operatorname{see}(2.21)), \varrho_{0} \in H^{2}(\Omega), 0<m$ $\leqq \varrho_{0}(x) \leqq M$ in $\bar{\Omega},\left[\theta_{0}-\bar{\theta}(0)\right] \in H_{0}^{1}(\Omega), \dot{\omega}_{0} \in H_{0}^{1}(\Omega)$ (see $\left.(2.22)\right), 0<\theta_{3} \leqq \theta_{0}(x) \leqq \theta_{4}$ in $\bar{\Omega},(\bar{u}, \bar{\theta}) \in L_{\mathrm{loc}}^{2}\left(\mathbb{R}^{+} ; H^{3}(\Omega)\right),\left(\bar{u}_{t}, \bar{\theta}_{t}\right) \in L_{\mathrm{loc}}^{2}\left(\mathbb{R}^{+} ; H^{2}(\Omega)\right),\left(\bar{u}_{t t}, \bar{\theta}_{t t}\right) \in L_{\mathrm{loc}}^{2}\left(\overline{\mathbb{R}}^{+} ; L^{2}(\Omega)\right)$, $\bar{u} \cdot \bar{n} \geqq 0$ on $\Sigma_{\infty}$. Then there exists $T^{*}>0$ small enough, $(u, \theta) \in L^{2}\left(0, T^{*} ; H^{3}(\Omega)\right)$, $\left(u_{t}, \theta_{t}\right) \in L^{2}\left(0, T^{*} ; \quad H^{2}(\Omega)\right), \quad\left(u_{t t}, \theta_{t t}\right) \in L^{2}\left(0, T^{*} ; \quad L^{2}(\Omega)\right), \quad \theta(t, x)>0 \quad$ in $\bar{Q}_{T^{*}}$, $\varrho \in C^{0}\left(\left[0, T^{*}\right] ; H^{2}(\Omega)\right), \varrho_{t} \in C^{0}\left(\left[0, T^{*}\right] ; H^{1}(\Omega)\right), \varrho(t, x)>0$ in $\bar{Q}_{T^{*}}$, such that $(u, \varrho, \theta)$ is a solution of (1.17) in $Q_{T^{*}}$.

Remark 2.6. By looking at the proof of Theorem 2.5 , it is easily seen that if $(b, r)$ $\in L^{\infty}\left(\mathbb{R}^{+} ; H^{1}(\Omega)\right),\left(b_{t}, r_{t}\right) \in L^{\infty}\left(\mathbb{R}^{+} ; L^{2}(\Omega)\right),(\bar{u}, \bar{\theta}) \in L^{\infty}\left(\mathbb{R}^{+} ; H^{3}(\Omega)\right),\left(\bar{u}_{t}, \bar{\theta}_{t}\right)$ $\in L^{\infty}\left(\mathbb{R}^{+} ; H^{2}(\Omega)\right),\left(\bar{u}_{t t}, \bar{\theta}_{t t}\right) \in L^{\infty}\left(\mathbb{R}^{+} ; L^{2}(\Omega)\right)$ and if

$$
\begin{gathered}
\left\|u_{0}-\bar{u}(0)\right\|_{5 / 2}^{2}+\left\|\sigma_{0}\right\|_{2}^{2}+\left\|\theta_{0}-\bar{\theta}(0)\right\|_{5 / 2}^{2}+\left\|\dot{v}_{0}\right\|_{1}^{2}+\left\|\dot{\omega}_{0}\right\|_{1}^{2} \leqq D, \\
0<A_{1} \leqq \inf _{\Omega}\left(\sigma_{0}+\bar{\varrho}\right) \leqq \sup _{\Omega}\left(\sigma_{0}+\bar{\varrho}\right) \leqq A_{2}, \\
0<E_{1} \leqq \inf _{\Omega}\left(\omega_{0}+\bar{\theta}(0)\right) \leqq \sup _{\Omega}\left(\omega_{0}+\bar{\theta}(0)\right) \leqq E_{2},
\end{gathered}
$$

then the time $T^{*}$ depends only on $\Omega, \mu, v, \chi, \bar{Q}, p, c_{v}$, on the norms of $b, b_{t}, r, r_{t}, \bar{u}, \bar{u}_{t}$, $\bar{u}_{t t}, \bar{\theta}, \bar{\theta}_{t}, \bar{\theta}_{t t}$ in the spaces considered before, and on $D, A_{1}, A_{2}, E_{1}, E_{2}$.

Remark 2.7. It is also possible to see that the time $T^{*}$ depends indeed on the norms of $b, b_{t}, r, r_{t}, \bar{u}, \bar{u}_{t}, \bar{u}_{t t}, \bar{\theta}, \bar{\theta}_{t}, \bar{\theta}_{t t}$ in the spaces of functions obtained from the space considered in Remark 2.6 by replacing $L^{\infty}$ with $\mathscr{S}^{2}$, where $\mathscr{S}^{2}\left(\mathbb{R}^{+} ; X\right)$ is the subspace of $L_{\text {loc }}^{2}\left(\mathbb{R}^{+} ; X\right)$ endowed by the norm

$$
\|u\|_{\mathscr{S}^{2}\left(\mathbb{R}^{+} ; X\right)}^{2} \equiv \sup _{t \in \mathbb{R}^{+}}\left[\int_{t}^{t+1}\|u(s)\|_{X}^{2} d s\right] .
$$


One has only to change the definition of $R_{T}$ by requiring

$$
\begin{gathered}
{[\tilde{\sigma}]_{\infty ; 2 ; T} \leqq B_{1},} \\
{\left[\tilde{v}_{t}\right]_{\infty ; 1 ; T}^{2}+\left[\tilde{v}_{t}\right]_{2 ; 2 ; T}^{2}+\left[\tilde{\omega}_{t}\right]_{\infty ; 1 ; T}^{2}+\left[\tilde{\omega}_{t}\right]_{2 ; 2 ; T}^{2} \leqq B_{2},} \\
{[\tilde{v}]_{\infty ; 2 ; T}^{2}+[\tilde{v}]_{2 ; 3 ; T}^{2}+[\tilde{\omega}]_{\infty ; 2 ; T}^{2}+[\tilde{\omega}]_{2 ; 3 ; T}^{2} \leqq B_{3},} \\
{\left[\tilde{\sigma}_{t}\right]_{\infty ; 1 ; T} \leqq B_{4},}
\end{gathered}
$$

and then to choose successively the constants $B_{1}, B_{2}, B_{3}, B_{4}$. Observe also that if $u \in \mathscr{S}\left(\mathbb{R}^{+} ; X\right)$ and $u_{t} \in \mathscr{S}^{2}\left(\mathbb{R}^{+} ; Y\right)$, (here $X$ and $Y$ are Hilbert spaces, with $X$ continuously embedded in $Y)$, then $u \in C_{B}^{0}\left(\mathbb{R}^{+} ;[X, Y]_{1 / 2}\right)$, and the immersion is continuous.

Remark 2.8. One can easily see that the solution $(v, \varrho, \theta)$ to problem $(1.17)$ is unique, for instance, in the class of functions

$$
\begin{gathered}
\inf _{Q_{T}} \varrho>0, \varrho \in L^{\infty}\left(Q_{T}\right), \nabla \varrho \in L^{4}\left(0, T ; L^{6}(\Omega)\right), \\
v \in L^{\infty}\left(Q_{T}\right), D v \in L^{4}\left(0, T ; L^{6}(\Omega)\right) \cap L^{2}\left(0, T ; L^{\infty}(\Omega)\right), v_{t} \in L^{2}\left(0, T ; L^{3}(\Omega)\right), \\
\inf _{Q_{T}} \theta>0, \theta \in L^{\infty}\left(Q_{T}\right), \nabla \theta \in L^{4}\left(0, T ; L^{6}(\Omega)\right) \cap L^{2}\left(0, T ; L^{\infty}(\Omega)\right), \\
\theta_{t} \in L^{2}\left(0, T ; L^{3}(\Omega)\right),
\end{gathered}
$$

under the assumptions $p \in C^{2}, c_{v} \in C^{1}, c_{v}>0$ for $\varrho>0$ and $\theta>0, b \in L^{2}\left(0, T ; L^{3}(\Omega)\right)$, $r \in L^{2}\left(0, T ; L^{3}(\Omega)\right), \partial \Omega \in C^{1}$. One obtains the same results also if $\mu, v$, and $\chi$ are not constant; it is sufficient to have $\mu, v$, and $\chi$ in $C^{1}$. Hence the fixed point constructed in Theorem 2.5 is unique. The proof of this uniqueness result can be obtained by proceeding as in $[29,31$, Sect. 3], and using the estimate

$$
\begin{gathered}
\int_{\Omega}\left(\left|\nabla \varrho_{1}^{*}\right|+\left|\nabla \theta_{1}^{*}\right|+\left|D v_{1}^{*}\right|\right)(|u|+|\gamma|)(|D u|+|\nabla \gamma|) \\
\leqq \varepsilon\|D u(t)\|_{0}^{2}+\varepsilon\|\nabla \gamma(t)\|_{0}^{2}+c \varepsilon^{-3}\left(\left\|\nabla \varrho_{1}^{*}(t)\right\|_{L^{6}(\Omega)}^{4}+\left\|\nabla \theta_{1}^{*}(t)\right\|_{L^{6}(\Omega)}^{4}\right. \\
\left.+\left\|D v_{1}^{*}(t)\right\|_{L^{6}(\Omega)}^{4}\right)\left(\|u(t)\|_{0}^{2}+\|\gamma(t)\|_{0}^{2}\right) .
\end{gathered}
$$

[Here $u \equiv u_{1}^{*}-u_{2}^{*}, \gamma \equiv \theta_{1}^{*}-\theta_{2}^{*}$, and $\left(u_{1}^{*}, \varrho_{1}^{*}, \theta_{1}^{*}\right),\left(u_{2}^{*}, \varrho_{2}^{*}, \theta_{2}^{*}\right)$ are two solutions of (1.17).]

\section{B. Case with an Inflow}

If we don't assume that $\bar{u} \cdot \bar{n} \geqq 0$ on $\Sigma_{T}$, then we need to change the proof of the local existence theorem. In fact in this case the solution of Eq. (2.13) can be determined only if we require in addition

$$
\left.\sigma_{\mid S_{1}}=\sigma_{1} \quad \text { on } \quad\right] 0, T\left[\times S_{1} .\right.
$$

Moreover, we suppose that $S_{1}$ is a closed surface (hence on $[0, T] \times S_{1}$ one has $-\bar{u} \cdot \bar{n} \geqq a_{0}>0, a_{0}$ a suitable constant which for simplicity we suppose to be less then 1).

Lemma 2.9. Let $\partial \Omega \in C^{2}, \tilde{u} \in L^{2}\left(0, T ; H^{3}(\Omega)\right), \tilde{u}_{t} \in L^{2}\left(0, T ; H^{2}(\Omega)\right), \sigma_{0} \in H^{2}(\Omega)$ and $\sigma_{1} \in L^{\infty}\left(0, T ; H^{2}\left(S_{1}\right)\right),\left(\sigma_{1}\right)_{t} \in L^{\infty}\left(0, T ; H^{1}\left(S_{1}\right)\right),\left(\sigma_{1}\right)_{t t} \in L^{\infty}\left(0, T ; L^{2}\left(S_{1}\right)\right)$. Suppose 
that $\tilde{u}, \sigma_{0}$, and $\sigma_{1}$ satisfy the compatibility conditions (2.26) and (2.27). Then there exists a unique solution of (2.13) satisfying $\sigma_{\mid S_{1}}=\sigma_{1}$, and such that $\sigma \in L^{\infty}\left(0, T ; H^{2}(\Omega)\right)$ with

$$
\begin{aligned}
{[\sigma]_{\infty ; 2 ; T}^{2} \leqq } & \left(\left\|\sigma_{0}\right\|_{2}^{2}+c T^{1 / 2}[\tilde{u}]_{2 ; 3 ; T}^{2}\right. \\
& \left.+\int_{0}^{T} Z\right) \exp \left(c[\tilde{u}]_{1 ; 3 ; T}+T^{1 / 2}\right)
\end{aligned}
$$

(see (2.28) for the definition of $Z$ ). If in addition $\tilde{u} \in L^{\infty}\left(0, T ; H^{2}(\Omega)\right.$ ), then $\sigma_{t} \in L^{\infty}\left(0, T ; H^{1}(\Omega)\right)$ and

$$
\left[\sigma_{t}\right]_{\infty ; 1 ; T} \leqq c_{6}[\tilde{u}]_{\infty ; 2 ; T}\left([\sigma]_{\infty ; 2 ; T}+1\right) .
$$

Proof. The existence of a solution follows from the theory of characteristics (see for instance Judovič [10]). If we divide $\bar{Q}_{T}$ into two subsets

$$
\begin{aligned}
& Q_{1} \equiv\left\{(t, x) \in \bar{Q}_{T} \mid \text { the characteristic passing for }(t, x)\right. \\
& \text { starts at } t=0 \text { on } \Omega\}, \\
& Q_{2} \equiv\left\{(t, x) \in \bar{Q}_{T} \mid \text { the characteristic passing for }(t, x)\right. \\
& \text { intersects } \left.\Sigma_{T} \text { for } 0 \leqq t^{*}=t^{*}(t, x) \leqq t\right\},
\end{aligned}
$$

then the solution $\sigma$ is expressed by (2.16) in $Q_{1}$ and by

$$
\begin{aligned}
\sigma(t, x)= & -\bar{\varrho}+\left[\sigma_{1}\left(t^{*}(t, x), U\left(t^{*}(t, x), t, x\right)\right)+\bar{\varrho}\right] \\
& \cdot \exp \left[-\int_{t^{*}(t, x)}^{t} \operatorname{div} \tilde{u}(s, U(s, t, x)) d s\right]
\end{aligned}
$$

in $Q_{2}$. One can see by a direct computation that, if $\sigma_{0}$ and $\sigma_{1}$ satisfy the following compatibility conditions:

$$
\begin{aligned}
& \sigma_{1 \mid t=0}=\sigma_{0 \mid S_{1}}, \\
& {\frac{\partial \sigma_{1}}{\partial t}}_{\mid t=0}=\left[-\tilde{u}(0) \cdot \nabla \sigma_{0}-\sigma_{0} \operatorname{div} \tilde{u}(0)-\bar{\varrho} \operatorname{div} \tilde{u}(0)\right]_{\mid S_{1}},
\end{aligned}
$$

then $\sigma \in L^{\infty}\left(0, T ; H^{2}(\Omega)\right)$ (see for instance Judovič [10] for similar calculations).

For proving that (2.23) holds one proceeds as in Lemma 2.4, but now the boundary terms arising from the integration by parts cannot be omitted. More precisely one needs to estimate

$$
-\int_{S_{1}} \bar{u} \cdot \bar{n}\left(\sigma_{1}^{2}+|\nabla \sigma|^{2}+\left|D^{2} \sigma\right|^{2}\right) .
$$

Hence one utilizes Eq. (2.13) $)_{1}$ restricted on $S_{1}$, and by proceeding as in Fiszdon and Zajaczkowski [4-6], after some long but straightforward calculations one gets

$$
\begin{aligned}
- & \int_{S_{1}} \bar{u} \cdot \bar{n}\left(\sigma_{1}^{2}+|\nabla \sigma|^{2}+\left|D^{2} \sigma\right|^{2}\right) \\
\leqq & c\left(1 / a_{0}\right)\left(1+\|\tilde{u}\|_{L^{\infty}\left(S_{1}\right)}^{2}\right)\left(1+\left|\sigma_{1}\right|_{2,0, S_{1}}^{2}\right) \\
& \cdot\left(1+\|\tilde{u}\|_{2}^{2}+\left\|\tilde{u}_{t}\right\|_{1}^{2}\right)\left(1+\|\tilde{u}\|_{5 / 2+\varepsilon}^{2}+\left\|\tilde{u}_{t}\right\|_{3 / 2+\varepsilon}^{2}\right) \equiv Z
\end{aligned}
$$

(for any $0<\varepsilon<1 / 2$ ). To obtain this estimate one uses the immersions

$$
H^{1}(\Omega) \hookrightarrow H^{1 / 2}\left(S_{1}\right) \hookrightarrow L^{4}\left(S_{1}\right), \quad H^{1 / 2+\varepsilon}(\Omega) \hookrightarrow L^{2}\left(S_{1}\right) .
$$


Hence we have

$$
\frac{d}{d t}\|\sigma\|_{2}^{2} \leqq c\|\tilde{u}\|_{3}\|\sigma\|_{2}^{2}+c\|\operatorname{div} \tilde{u}\|_{2}\|\sigma\|_{2}+Z
$$

But

$$
c\|\operatorname{div} \tilde{u}\|_{2}\|\sigma\|_{2} \leqq c T^{1 / 2}\|\tilde{u}\|_{3}^{2}+T^{-1 / 2}\|\sigma\|_{2}^{2},
$$

hence by Gronwall's lemma

$$
\begin{aligned}
\|\sigma\|_{2}^{2} \leqq & {\left[\left\|\sigma_{0}\right\|_{2}^{2}+c T^{1 / 2}[\tilde{u}]_{2 ; 3 ; T}^{2}+c \int_{0}^{T} Z\right] } \\
& \cdot \exp \left(c[\tilde{u}]_{1 ; 3 ; T}+T^{1 / 2}\right) .
\end{aligned}
$$

One can observe now that if $\tilde{u}_{t t} \in L^{2}\left(0, T ; L^{2}(\Omega)\right)$, then

$$
\begin{aligned}
\int_{0}^{T} Z \leqq & c\left(1 / a_{0}\right)\left(1+\|\tilde{u}\|_{L^{\infty}\left(\Sigma_{T}\right)}^{2}\right)\left(1+\sup _{[0, T]}\left|\sigma_{1}\right|_{2,0, S_{1}}^{2}\right) \\
& \cdot\left(1+[\tilde{u}]_{\infty ; 2 ; T}^{2}+\left[\tilde{u}_{t}\right]_{\infty ; 1 ; T}^{2}\right) \\
& \cdot\left[T+c(\varepsilon) T^{1-2 \varepsilon}\left([\tilde{u}]_{2 ; 3 ; T}^{2}+\left[\tilde{u}_{t}\right]_{2 ; 2 ; T}^{2}+\|\tilde{u}(0)\|_{5 / 2}^{2}\right)\right. \\
+ & \left.c(\varepsilon) T^{1 / 2-\varepsilon}\left(\left[\tilde{u}_{t}\right]_{2 ; 2 ; T}^{2}+\left[\tilde{u}_{t t}\right]_{2 ; 0 ; T}^{2}+\left\|\tilde{u}_{t}(0)\right\|_{1}^{2}\right)\right],
\end{aligned}
$$

where one uses these interpolation results:

$$
\begin{gathered}
{[\tilde{u}]_{1 / \varepsilon ; 5 / 2+\varepsilon ; T}^{2} \leqq c(\varepsilon)\left([\tilde{u}]_{2 ; 3 ; T}^{2}+\left[\tilde{u}_{t}\right]_{2 ; 2 ; T}^{2}+\|\tilde{u}(0)\|_{5 / 2}^{2}\right),} \\
{\left[\tilde{u}_{t}\right]_{q ; 3 / 2+\varepsilon ; T}^{2} \leqq c(\varepsilon)\left(\left[\tilde{u}_{t}\right]_{2 ; 2 ; T}^{2}+\left[\tilde{u}_{t t}\right]_{2 ; 0 ; T}^{2}+\left\|\tilde{u}_{t}(0)\right\|_{1}^{2}\right),} \\
q=\frac{4}{1+2 \varepsilon},
\end{gathered}
$$

where $c=c(\varepsilon)$ does not depend on $T$ (see, for instance, Secchi and Valli [22, Corollary 3.10], where similar estimates are obtained).

Hence one can prove the existence of a local solution also in the case with an inflow, by changing the definition of the set $R_{T}$ in the sense that now one needs $\tilde{v}_{t t} \in L^{2}\left(0, T ; L^{2}(\Omega)\right.$ ) (and the norm in this space is estimated by $B_{1}$ ), and $\tilde{v}_{t}(0)=\dot{v}_{0}$ [see (2.21)]. One can prove also in this situation that $R_{T} \neq \emptyset$; in fact construct a function $v^{*}$ as in Sect. A, by choosing now $H$ in such a way that $H \in L^{2}\left(\mathbb{R}^{+} ; H^{1}(\Omega)\right)$, $H_{t} \in L^{2}\left(\mathbb{R}^{+} ; L^{2}(\Omega)\right)$ and $H(0)=-\Delta\left(u_{0}-\bar{u}(0)\right)+\dot{v}_{0}$. By choosing $B_{1}$ large enough, one gets again $R_{T} \neq \emptyset$ for $T$ sufficiently small.

Hence one obtains a local existence theorem under the same assumptions of Theorem 2.5, only requiring further that $\sigma_{1} \in L_{\text {loc }}^{\infty}\left(\mathbb{R}^{+} ; H^{2}\left(S_{1}\right)\right)$, $\left(\sigma_{1}\right)_{t} \in L_{\mathrm{loc}}^{\infty}\left(\mathbb{R}^{+} ; H^{1}\left(S_{1}\right)\right),\left(\sigma_{1}\right)_{t t} \in L_{\mathrm{loc}}^{\infty}\left(\mathbb{R}^{+} ; L^{2}\left(S_{1}\right)\right)$, and that $\sigma_{1}, \sigma_{0}$, and $\tilde{u}$ satisfy the compatibility conditions (2.26) and (2.27).

\section{Global Existence. The Case Without an Inflow}

At first we shall obtain some a priori estimates for the solutions of the following system of equations 


$$
\begin{aligned}
& v_{t}+\bar{A} v+p_{1} \nabla \sigma+p_{2} \nabla \omega=f \quad \text { in } \quad Q_{T}, \\
& v=0 \text { on } \Sigma_{T}, \\
& v(0)=v_{0} \quad \text { in } \Omega \text {, }
\end{aligned}
$$

where $\bar{A} \equiv-\bar{\mu} \Delta-\bar{v} \nabla \operatorname{div}$ and $\bar{\mu} \equiv \mu / \bar{\varrho}, v \equiv v / \bar{\varrho}$;

$$
\begin{aligned}
\sigma_{t}+(v+\tilde{u}) \cdot \nabla \sigma+\tilde{\varrho} \operatorname{div} v & =h \quad \text { in } \quad Q_{T}, \\
\sigma(0) & =\sigma_{0} \quad \text { in } \quad \Omega ; \\
\omega_{t}-\bar{\chi} \Delta \omega+\theta_{1} p_{3} \operatorname{div} v & =g \quad \text { in } \quad Q_{T}, \\
\omega & =0 \quad \text { on } \quad \Sigma_{T}, \\
\omega(0) & =\omega_{0} \quad \text { in } \Omega,
\end{aligned}
$$

where

$$
\begin{gathered}
\theta_{1} \equiv \inf _{\Sigma_{\infty}} \bar{\theta}>0, \\
\bar{\chi} \equiv \frac{\chi}{\bar{\varrho} c_{v}\left(\bar{\varrho}, \theta_{1}\right)}, \\
p_{1} \equiv \frac{p_{\varrho}\left(\bar{\varrho}, \theta_{1}\right)}{\bar{\varrho}}>0, \quad p_{2} \equiv \frac{p_{\theta}\left(\bar{\varrho}, \theta_{1}\right)}{\bar{\varrho}}, \quad p_{3} \equiv \frac{p_{2}}{c_{v}\left(\bar{\varrho}, \theta_{1}\right)},
\end{gathered}
$$

and consequently $p_{2} / p_{3}=c_{v}\left(\bar{\varrho}, \theta_{1}\right)>0$, and

$$
f \equiv f^{1}+f^{2}+f^{3}
$$

where

$$
\begin{gathered}
f^{1} \equiv\left(p_{1}-\frac{p_{\varrho}(\sigma+\bar{\varrho}, \omega+\bar{\theta})}{\sigma+\bar{\varrho}}\right) \nabla \sigma, \quad f^{2} \equiv\left(p_{2}-\frac{p_{\theta}(\sigma+\bar{\varrho}, \omega+\bar{\theta})}{\sigma+\bar{\varrho}}\right) \nabla(\omega+\bar{\theta}) \\
f^{3} \equiv-(v+\bar{u}) \cdot \nabla(v+\bar{u})+\frac{\sigma}{\sigma+\bar{\varrho}} \bar{A} v+b-\bar{u}_{t}-\frac{1}{\sigma+\bar{\varrho}} A \bar{u} \\
h \equiv-\sigma \operatorname{div} v-(\sigma+\bar{\varrho}) \operatorname{div} \bar{u} \\
g \equiv g^{1}+g^{2}+g^{3}
\end{gathered}
$$

where

$$
\begin{gathered}
g^{1} \equiv\left[\theta_{1} \frac{p_{\theta}\left(\bar{\varrho}, \theta_{1}\right)}{\bar{\varrho} c_{v}\left(\bar{\varrho}, \theta_{1}\right)}-\frac{\omega+\bar{\theta}}{\sigma+\bar{\varrho}} \frac{p_{\theta}(\sigma+\bar{\varrho}, \omega+\bar{\theta})}{c_{v}(\sigma+\bar{\varrho}, \omega+\bar{\theta})}\right] \\
\cdot \operatorname{div} v-\left[\bar{\chi}-\frac{\chi}{(\sigma+\bar{\varrho}) c_{v}(\sigma+\bar{\varrho}, \omega+\bar{\theta})}\right] \Delta \omega, \\
g^{2} \equiv \frac{\chi}{(\sigma+\bar{\varrho}) c_{v}(\sigma+\bar{\varrho}, \omega+\bar{\theta})} \Delta \bar{\theta}-\frac{\omega+\bar{\theta}}{\sigma+\bar{\varrho}} \frac{p_{\theta}(\sigma+\bar{\varrho}, \omega+\bar{\theta})}{c_{v}(\sigma+\bar{\varrho}, \omega+\bar{\theta})} \operatorname{div} \bar{u},
\end{gathered}
$$




$$
\begin{aligned}
g^{3} \equiv & -\bar{\theta}_{t}-(v+\bar{u}) \cdot \nabla(\omega+\bar{\theta})+\frac{1}{(\sigma+\bar{\varrho}) c_{v}(\sigma+\bar{\varrho}, \omega+\bar{\theta})} \\
& \cdot\left\{\frac{\mu}{2} \sum_{i, k}\left[D_{i}(v+\bar{u})^{k}+D_{k}(v+\bar{u})^{i}\right]^{2}+(v-\mu)[\operatorname{div}(v+\bar{u})]^{2}\right\} \\
& +\frac{r}{c_{v}(\sigma+\bar{\varrho}, \omega+\bar{\theta})} .
\end{aligned}
$$

We shall assume that $\bar{u} \cdot \bar{n} \geqq 0$ on $\Sigma_{T}$, that is we don't consider the case of an inflow. Moreover, we assume that $\partial \Omega \in C^{3}$, and that

$$
\bar{\varrho} / 4 \leqq \sigma(t, x)+\bar{\varrho} \leqq 3 \bar{\varrho}, \quad \theta_{1} / 4 \leqq \omega(t, x)+\bar{\theta}(t, x) \leqq 3 \theta_{2}
$$

in $\bar{Q}_{T}, 0<T \leqq \infty$. (Here $\left.\theta_{2} \equiv \sup _{\Sigma_{\infty}} \bar{\theta}\right)$.

From the conditions $c_{v} \in C^{1}, c_{v}>0$ for $\varrho>0$ and $\theta>0$, we obtain thus $0<c_{1}^{*} \leqq c_{v}(\sigma+\bar{\varrho}, \omega+\bar{\theta}) \leqq c_{2}^{*}\left[\right.$ where $c_{1}^{*}$ and $c_{2}^{*}$ are defined as in Sect. 2, and the set $\Lambda_{1}$ is constructed by means of the constants appearing in (3.7)]. Finally, from now on in this section each constant will depend (at most) on $\Omega, \mu, v, \chi, \bar{\varrho}, \theta_{1}, \theta_{2}, p_{1}, p_{2}$, $p_{3}, c_{1}^{*}, c_{2}^{*}$ and on the norms of $p$ in $C^{2}\left(\Lambda_{1}\right)$ and of $c_{v}$ in $C^{1}\left(\Lambda_{1}\right)$.

We remark again that in this section we shall indicate any equivalent norm in $H^{s}(\Omega)$ with the same symbol $\|\cdot\|_{s}(s \geqq-1)$.

Lemma 3.1. Let $v, \sigma, \omega$ be a sufficiently smooth solution of (3.1)-(3.3), and $\varepsilon_{3}$ be a sufficiently small parameter, then

$$
\begin{aligned}
& \frac{d}{d t}\left(\|v\|_{1}^{2}+\left\|v_{t}\right\|_{1}^{2}+\|\omega\|_{1}^{2}+\left\|\omega_{t}\right\|_{1}^{2}+\|\sigma\|_{0}^{2}+\left\|\sigma_{t}\right\|_{0}^{2}\right) \\
& \quad+\|v\|_{2}^{2}+\left\|v_{t}\right\|_{2}^{2}+\|\omega\|_{2}^{2}+\left\|\omega_{t}\right\|_{2}^{2}+\|\sigma\|_{1}^{2}+\left\|\sigma_{t}\right\|_{1}^{2} \\
& \quad \leqq \varepsilon_{3}\|v\|_{3}^{2}+c\left(\varepsilon_{3}\right) Y_{1}
\end{aligned}
$$

where

$$
\begin{aligned}
Y_{1} \equiv & \|f\|_{0}^{2}+\left\|f_{t}\right\|_{0}^{2}+\|g\|_{0}^{2}+\left\|g_{t}\right\|_{0}^{2}+|h|_{1,0}^{2}+\|\sigma\|_{2}^{4} \\
& +\|\bar{u}\|_{3}\left(\|\sigma\|_{0}^{2}+\left\|\sigma_{t}\right\|_{0}^{2}\right)+\left\|\bar{u}_{t}\right\|_{1}\|\sigma\|_{2}\left\|\sigma_{t}\right\|_{0}+\|v\|_{2}^{2}\|\sigma\|_{2}^{2} \\
& +\|\bar{u}\|_{2}^{2}\|\sigma\|_{2}^{2}+\left|\int_{0}^{t} \int_{S_{2}} \bar{u} \cdot \bar{n}\right|^{2} .
\end{aligned}
$$

Proof. Multiply (3.1) by $v,(3.2)$ by $\left(p_{1} / \varrho\right) \sigma$, and (3.3) by $\left(p_{2} / p_{3} \theta_{1}\right) \omega$, integrate over $\Omega$ and add these expressions. Taking into account that

$$
\int_{\Omega} p_{1} \nabla \sigma \cdot v=-\int_{\Omega} p_{1} \sigma \operatorname{div} v, \quad \int_{\Omega} p_{2} \nabla \omega \cdot v=-\int_{\Omega} p_{2} \omega \operatorname{div} v,
$$

and that

$$
\begin{aligned}
& -\frac{p_{1}}{\varrho} \int_{\Omega}[(v+\bar{u}) \cdot \nabla \sigma] \sigma=-\frac{p_{1}}{2 \varrho} \int_{\Omega}(v+\bar{u}) \cdot \nabla\left(\sigma^{2}\right) \\
& \leqq \frac{p_{1}}{2 \varrho} \int_{\Omega}|\operatorname{div}(v+\bar{u})| \sigma^{2} \leqq \varepsilon_{1}\|v\|_{3}^{2}+\frac{c}{\varepsilon_{1}}\|\sigma\|_{0}^{4}+c\|\bar{u}\|_{3}\|\sigma\|_{0}^{2}
\end{aligned}
$$


at last one gets

$$
\frac{d}{d t}\left(\|v\|_{0}^{2}+\|\omega\|_{0}^{2}+\|\sigma\|_{0}^{2}\right)+\|v\|_{1}^{2}+\|\omega\|_{1}^{2} \leqq c X_{1}+\varepsilon_{1}\|v\|_{3}^{2}+\varepsilon_{1}\|\sigma\|_{0}^{2},
$$

where

$$
X_{1} \equiv\|f\|_{-1}^{2}+\|g\|_{-1}^{2}+\frac{1}{\varepsilon_{1}}\|h\|_{0}^{2}+\frac{1}{\varepsilon_{1}}\|\sigma\|_{0}^{4}+\|\tilde{u}\|_{3}\|\sigma\|_{0}^{2} .
$$

Similarly to (3.9) one has

$$
\begin{aligned}
& \frac{d}{d t}\left(\left\|v_{t}\right\|_{0}^{2}+\left\|\omega_{t}\right\|_{0}^{2}+\left\|\sigma_{t}\right\|_{0}^{2}\right)+\left\|v_{t}\right\|_{1}^{2}+\left\|\omega_{t}\right\|_{1}^{2} \\
& \quad \leqq c X_{2}+\varepsilon_{1}\|v\|_{3}^{2}+\varepsilon_{2}\left\|\sigma_{t}\right\|_{0}^{2}
\end{aligned}
$$

where

$$
\begin{aligned}
X_{2} \equiv & \left\|f_{t}\right\|_{-1}^{2}+\left\|g_{t}\right\|_{-1}^{2}+\frac{1}{\varepsilon_{2}}\left\|h_{t}\right\|_{0}^{2}+\frac{1}{\varepsilon_{1}}\left\|\sigma_{t}\right\|_{0}^{4}+\left\|\sigma_{t}\right\|_{0}^{2}\|\sigma\|_{2}^{2} \\
& +\|\bar{u}\|_{3}\left\|\sigma_{t}\right\|_{0}^{2}+\left\|\bar{u}_{t}\right\|_{1}\|\sigma\|_{2}\left\|\sigma_{t}\right\|_{0} .
\end{aligned}
$$

The only difference in obtaining (3.10) is the way of estimating the non-linear term $\left(p_{1} / \varrho\right) \int_{\Omega} \frac{\partial}{\partial t}[(v+\bar{u}) \cdot \nabla \sigma] \sigma_{t}$ (see, for instance [31, Lemma 4.2]).

Using the Stokes' problem

$$
\begin{aligned}
-\bar{\mu} \Delta v+p_{1} \nabla \sigma & =-v_{t}-p_{2} \nabla \omega+\bar{v} \nabla \operatorname{div} v+f \text { in } \Omega . \\
\operatorname{div} v & =\operatorname{div} v \quad \text { in } \Omega, \\
v_{\mid \partial \Omega} & =0 \text { on } \partial \Omega,
\end{aligned}
$$

one has (see for instance, $[3,7]$ )

$$
\|v\|_{2}^{2}+\|\sigma\|_{1}^{2} \leqq c\left(\left\|v_{t}\right\|_{0}^{2}+\|\omega\|_{1}^{2}+\|\operatorname{div} v\|_{1}^{2}+\|f\|_{0}^{2}\right)+c\left|\int_{0}^{t} \int_{S_{2}} \bar{u} \cdot \bar{n}\right|^{2},
$$

where the last term in the right-hand side appears in the case of outflow only. Indeed, for $\bar{u} \cdot \bar{n}_{\mid \partial \Omega}=0$ one has that $\int_{\Omega} \sigma=0$, so $\|\sigma\|_{0}^{2} \leqq c\|\nabla \sigma\|_{0}^{2}$. In the case of outflow (3.2) implies $\int_{\Omega} \sigma=-\int_{0}^{t} \int_{S_{2}} \bar{u} \cdot \bar{n}(\sigma+\varrho \bar{\varrho})$, so

$$
\|\sigma\|_{0}^{2} \leqq c\|\nabla \sigma\|_{0}^{2}+c\left|\int_{\Omega} \sigma\right|^{2} \leqq c\|\nabla \sigma\|_{0}^{2}+c\left|\int_{0}^{t} \int_{S_{2}} \bar{u} \cdot \bar{n}\right|^{2} .
$$

Directly from (3.2) $)_{1}$ one gets

$$
\left\|\sigma_{t}\right\|_{1}^{2} \leqq c\left(\|\operatorname{div} v\|_{1}^{2}+\|h\|_{1}^{2}+\|v\|_{2}^{2}\|\sigma\|_{2}^{2}+\|\bar{u}\|_{2}^{2}\|\sigma\|_{2}^{2}\right) .
$$


Using the interpolation inequality

$$
c\|\operatorname{div} v\|_{1}^{2} \leqq \varepsilon_{3}\|v\|_{3}^{2}+\frac{c}{\varepsilon_{3}}\|v\|_{1}^{2}, \quad 0<\varepsilon_{3}<1
$$

in (3.12) and (3.13), from (3.9), (3.10), (3.12), and (3.13) one gets

$$
\begin{aligned}
& \frac{d}{d t}\left(\frac{1}{\varepsilon_{3}}\|v\|_{0}^{2}+\left\|v_{t}\right\|_{0}^{2}+\frac{1}{\varepsilon_{3}}\|\omega\|_{0}^{2}+\left\|\omega_{t}\right\|_{0}^{2}+\frac{1}{\varepsilon_{3}}\|\sigma\|_{0}^{2}+\left\|\sigma_{t}\right\|_{0}^{2}\right) \\
& \quad+|v|_{2,1}^{2}+\|\omega\|_{1}^{2}+\left\|\omega_{t}\right\|_{1}^{2}+\|\sigma\|_{1}^{2}+\left\|\sigma_{t}\right\|_{1}^{2} \\
& \quad \leqq c X_{3}+\varepsilon_{3}\left(\|v\|_{3}^{2}+\|\sigma\|_{0}^{2}\right),
\end{aligned}
$$

where

$$
\begin{aligned}
X_{3} \equiv & \left(1 / \varepsilon_{3}\right) X_{1}+X_{2}+\|f\|_{0}^{2}+\|h\|_{1}^{2}+\left(\|v\|_{2}^{2}+\|\bar{u}\|_{2}^{2}\right)\|\sigma\|_{2}^{2} \\
& +\left|\int_{0}^{t} \int_{S_{2}} \bar{u} \cdot \bar{n}\right|^{2} .
\end{aligned}
$$

For obtaining (3.15) one has chosen $\varepsilon_{2}$ small enough and $\varepsilon_{1}=\varepsilon_{3}^{2}$.

Multiplying (3.1) and (3.3) by $\bar{A} v$ and $-\Delta \omega$, respectively, differentiating (3.1) and (3.3) with respect to $t$, multiplying by $\bar{A} v_{t}$ and $-\Delta \omega_{t}$, respectively, adding these results and integrating over $\Omega$, one gets

$$
\begin{gathered}
\frac{d}{d t}\left(\|D v\|_{0}^{2}+\|\operatorname{div} v\|_{0}^{2}+\|\nabla \omega\|_{0}^{2}+\left\|D v_{t}\right\|_{0}^{2}+\left\|\operatorname{div} v_{t}\right\|_{0}^{2}+\left\|\nabla \omega_{t}\right\|_{0}^{2}\right) \\
\quad+\|v\|_{2}^{2}+\|\omega\|_{2}^{2}+\left\|v_{t}\right\|_{2}^{2}+\left\|\omega_{t}\right\|_{2}^{2} \\
\leqq c X_{4}+c\left(\|\sigma\|_{1}^{2}+\left\|\sigma_{t}\right\|_{1}^{2}+\|v\|_{1}^{2}+\left\|v_{t}\right\|_{1}^{2}+\|\omega\|_{1}^{2}+\left\|\omega_{t}\right\|_{1}^{2}\right),
\end{gathered}
$$

where

$$
X_{4} \equiv\|f\|_{0}^{2}+\|g\|_{0}^{2}+\left\|f_{t}\right\|_{0}^{2}+\left\|g_{t}\right\|_{0}^{2} .
$$

By adding now (3.16) and (3.15), and choosing $\varepsilon_{3}$ small enough, one obtains (3.8).

Introduce now a partition of unity $\left(\left\{\tilde{\Omega}_{i}\right\},\left\{\xi_{i}\right\}\right)$. Let $\widetilde{\Omega}$ be one of the $\widetilde{\Omega}_{i}$ 's, and $\xi(x)$ the function relative to $\widetilde{\Omega}$. For subdomains $\widetilde{\Omega}$ such that $\widetilde{\Omega} \cap \partial \Omega \neq \emptyset$ new coordinates $y=T(x)$ such that $\left\{y^{3}=0\right\}=T(\tilde{\Omega} \cap \partial \Omega)$ are introduced. For simplicity set $y^{\alpha}=\tau^{\alpha}$ $(\alpha=1,2), y^{3}=n$. Moreover, define $\hat{f}(y) \equiv f\left(T^{-1}(y)\right), \hat{\Omega} \equiv T(\widetilde{\Omega} \cap \Omega), \tilde{u} \equiv \hat{u} \hat{\xi}, \hat{\nabla} \equiv y_{x} \nabla$, where $\nabla \equiv \nabla_{y}$, and set $\lambda \equiv \operatorname{diam} \tilde{\Omega}$. Later on the dependence of the constants on $\lambda$ will be explicitly pointed out.

Equations (3.1), (3.2) have now the following form

$$
\begin{gathered}
\tilde{v}_{t}+\bar{A} \tilde{v}+p_{1} \nabla \tilde{\sigma}+p_{2} \nabla \tilde{\omega}=\tilde{f}+k_{1}, \\
\tilde{\sigma}_{t}+\hat{u} \cdot \hat{\nabla} \tilde{\sigma}+\varrho \operatorname{div} \tilde{v}=\tilde{h}+k_{2},
\end{gathered}
$$

where $k_{1}$ and $k_{2}$ are defined in (3.20)-(3.23), and for simplicity we write $u \equiv v+\bar{u}$. By taking the gradient of (3.18) multiplied by $(\bar{\mu}+\bar{v}) / \bar{\varrho}$ and adding the result to (3.17) 
one has

$$
\begin{array}{r}
\frac{\bar{\mu}+\bar{v}}{\varrho} \nabla \tilde{\sigma}_{t}+p_{1} \nabla \tilde{\sigma}=\bar{\mu}(\Delta \tilde{v}-\nabla \operatorname{div} \tilde{v})-\tilde{v}_{t}-p_{2} \nabla \tilde{\omega} \\
-\frac{\bar{\mu}+\bar{v}}{\varrho} \nabla(\hat{u} \cdot \hat{\nabla} \tilde{\sigma})+\tilde{f}+\frac{\bar{\mu}+\bar{v}}{\varrho} \nabla \tilde{h}+k_{4},
\end{array}
$$

where $k_{4} \equiv \frac{\bar{\mu}+\bar{v}}{\bar{\varrho}} \nabla k_{2}+k_{1}, k_{i} \equiv k_{i 1}+k_{i 2}, i=1,2$, and

$$
\begin{gathered}
k_{11} \equiv p_{1}(\nabla-\hat{\nabla}) \tilde{\sigma}+p_{2}(\nabla-\hat{\nabla}) \tilde{\omega}-\bar{\mu}(\Delta-\hat{\Delta}) \tilde{v}-\bar{v}(\nabla \operatorname{div}-\hat{\nabla} \operatorname{div}) \tilde{v} \\
k_{12} \equiv p_{1} \hat{\sigma} \hat{\nabla} \hat{\xi}+p_{2} \hat{\omega} \hat{\nabla} \hat{\xi}-\bar{\mu}\left(2 \hat{\nabla_{k}} \hat{v} \hat{\nabla_{k}} \hat{\xi}+\hat{v} \hat{\Delta} \hat{\xi}\right) \\
-\bar{v}\left(\operatorname{div} \hat{v} \hat{\nabla} \hat{\xi}+\hat{\nabla} \hat{v}^{k} \hat{\nabla_{k}} \hat{\xi}+\hat{v}^{k} \hat{\nabla} \hat{\nabla}_{k} \hat{\xi}\right) \\
k_{21} \equiv \bar{\varrho}(\operatorname{div}-\operatorname{div}) \tilde{v} \\
k_{22} \equiv \hat{\sigma} \hat{u} \hat{\nabla} \hat{\xi}+\varrho \hat{v} \hat{\nabla} \hat{\xi} .
\end{gathered}
$$

For interior subdomains $\tilde{\Omega}$, the transformation $T$ is the identity, $\hat{\nabla}=\nabla_{y}=\nabla_{x}, \hat{f}=f$ and $k_{i 1}=0, i=1,2$.

Lemma 3.2. For a sufficiently smooth solution of (3.1)-(3.3) and by taking the parameter $\lambda$ small enough, one has

$$
\begin{aligned}
& \frac{d}{d t}\left(\left\|v_{\tau \tau}\right\|_{0}^{2}+\left\|D^{2} \sigma\right\|_{0}^{2}\right)+\left\|D^{2} v_{\tau}\right\|_{0}^{2}+\left\|D^{2} \sigma\right\|_{0}^{2}+\left\|D^{2} \operatorname{div} v\right\|_{0}^{2} \\
& \quad \leqq c \sqrt{\lambda}\|v\|_{3}^{2}+c(\lambda)\left(\left\|\left.v\right|_{2,1} ^{2}+\right\| \omega\left\|_{2}^{2}+\right\| \sigma \|_{1}^{2}\right)+c(\lambda) Y_{2}
\end{aligned}
$$

where

$$
Y_{2} \equiv\|f\|_{1}^{2}+\|h\|_{2}^{2}+\left(\|v\|_{2}^{2}+\|\bar{u}\|_{2}^{2}\right)\|\sigma\|_{2}^{2}+\left(\|v\|_{3}+\|\bar{u}\|_{3}\right)\|\sigma\|_{2}^{2},
$$

and $v_{\tau}$ means that we consider the tangential derivative of $v$.

Proof. Differentiating (3.17), (3.18) twice with respect to $\tau$, multiplying by $\tilde{v}_{\tau \tau}$, $\left(p_{1} / \bar{\varrho}\right) \tilde{\sigma}_{\tau \tau}$, respectively, integrating over $\hat{\Omega}$ and summing we get, by assuming $\lambda \equiv \operatorname{diam} \tilde{\Omega}$ small enough and $0<\varepsilon_{4}<1$ :

$$
\begin{aligned}
& \frac{d}{d t}\left(\left\|\tilde{v}_{\tau \tau}\right\|_{0}^{2}+\left\|\tilde{\sigma}_{\tau \tau}\right\|_{0}^{2}\right)+\left\|\tilde{v}_{y \tau \tau}\right\|_{0}^{2}+\left\|\operatorname{div} \tilde{v}_{\tau \tau}\right\|_{0}^{2} \\
& \quad \leqq c \lambda\left(\left\|\tilde{\sigma}_{y \tau}\right\|_{0}^{2}+\frac{1}{\varepsilon_{4}}\left\|\tilde{v}_{\tau y y}\right\|_{0}^{2}\right)+\varepsilon_{4}\left\|\tilde{\sigma}_{\tau \tau}\right\|_{0}^{2}+c(\lambda) X_{5},
\end{aligned}
$$

where

$$
\begin{aligned}
X_{5} \equiv & \|\sigma\|_{1}^{2}+\frac{1}{\varepsilon_{4}}\|v\|_{2}^{2}+\|\omega\|_{2}^{2}+\|f\|_{1}^{2}+\frac{1}{\varepsilon_{4}}\|h\|_{2}^{2} \\
& +\frac{1}{\varepsilon_{4}}\left(\|v\|_{2}^{2}+\|\bar{u}\|_{2}^{2}\right)\|\sigma\|_{2}^{2}+\left(\|v\|_{3}+\|\bar{u}\|_{3}\right)\|\sigma\|_{2}^{2}
\end{aligned}
$$


Differentiating the third component of (3.19) with respect to $\tau$, multiplying by $\tilde{\sigma}_{n \tau}$ and integrating over $\hat{\Omega}$, we have

$$
\frac{d}{d t}\left\|\tilde{\sigma}_{n \tau}\right\|_{0}^{2}+\left\|\tilde{\sigma}_{n \tau}\right\|_{0}^{2} \leqq c\left\|\tilde{v}_{y \tau \tau}\right\|_{0}^{2}+c \lambda\left(\left\|\tilde{\sigma}_{y \tau}\right\|_{0}^{2}+\left\|\tilde{v}_{y y \tau}\right\|_{0}^{2}\right)+c(\lambda) X_{6},
$$

where

$$
X_{6} \equiv\left\|v_{t}\right\|_{1}^{2}+X_{5} \text {. }
$$

Equation (3.17) can be written in the form

$$
(\tilde{\mu}+\bar{v}) \nabla \operatorname{div} \tilde{v}=-\tilde{\mu}(\Delta \tilde{v}-\nabla \operatorname{div} \tilde{v})+p_{1} \nabla \tilde{\sigma}+p_{2} \nabla \tilde{\omega}+\tilde{v}_{t}-\tilde{f}-k_{1} .
$$

Differentiating the third component of (3.27) with respect to $\tau$ one has

$$
\begin{aligned}
& \left\|\operatorname{div} \tilde{v}_{n \tau}\right\|_{0}^{2} \\
& \quad \leqq c\left(\left\|\tilde{v}_{y \tau \tau}\right\|_{0}^{2}+\left\|\tilde{\sigma}_{n \tau}\right\|_{0}^{2}\right)+c \lambda\left(\left\|\tilde{\sigma}_{y \tau}\right\|_{0}^{2}+\left\|\tilde{v}_{y y \tau}\right\|_{0}^{2}\right)+c(\lambda) X_{6} .
\end{aligned}
$$

From (3.26) and (3.28) one obtains

$$
\begin{aligned}
& \frac{d}{d t}\left\|\tilde{\sigma}_{n \tau}\right\|_{0}^{2}+\left\|\tilde{\sigma}_{n \tau}\right\|_{0}^{2}+\left\|\operatorname{div} \tilde{v}_{n \tau}\right\|_{0}^{2} \\
& \quad \leqq c\left\|\tilde{v}_{y \tau \tau}\right\|_{0}^{2}+c \lambda\left(\left\|\tilde{\sigma}_{y \tau}\right\|_{0}^{2}+\left\|\tilde{v}_{y y \tau}\right\|_{0}^{2}\right)+c(\lambda) X_{6} .
\end{aligned}
$$

Consider now the following Stokes' problem

$$
\begin{aligned}
-\bar{\mu} \Delta \tilde{v}+p_{1} \nabla \tilde{\sigma} & =-\tilde{v}_{t}-p_{2} \nabla \tilde{\omega}+\bar{v} \nabla \operatorname{div} \tilde{v}+\tilde{f}+k_{1} \text { in } \hat{\Omega}, \\
\operatorname{div} \tilde{v} & =\operatorname{div} \tilde{v} \quad \text { in } \hat{\Omega}, \\
\tilde{v}_{\mid \partial \hat{\Omega}} & =0 \text { on } \partial \hat{\Omega} .
\end{aligned}
$$

Differentiating (3.30) with respect to $\tau$ one gets

$$
\left\|\tilde{v}_{\tau y y}\right\|_{0}^{2}+\left\|\tilde{\sigma}_{y \tau}\right\|_{0}^{2} \leqq c\left\|\nabla \operatorname{div} \tilde{v}_{\tau}\right\|_{0}^{2}+c \lambda\left(\left\|\tilde{\sigma}_{y \tau}\right\|_{0}^{2}+\left\|\tilde{v}_{y y \tau}\right\|_{0}^{2}\right)+c(\lambda) X_{6} .
$$

From the normal derivatives of the third components of (3.19) and (3.27) we obtain

$$
\begin{aligned}
& \frac{d}{d t}\left\|\tilde{\sigma}_{n n}\right\|_{0}^{2}+\left\|\tilde{\sigma}_{n n}\right\|_{0}^{2}+\left\|\operatorname{div} \tilde{v}_{n n}\right\|_{0}^{2} \\
& \quad \leqq c\left\|\tilde{v}_{y n \tau}\right\|_{0}^{2}+c \lambda\left(\left\|\tilde{v}_{n y y}\right\|_{0}^{2}+\left\|\tilde{\sigma}_{n y}\right\|_{0}^{2}\right)+c(\lambda) X_{6} .
\end{aligned}
$$

At last from (3.25), (3.29), (3.31), and (3.32), for sufficiently small $\varepsilon_{4}$ and for $\lambda \leqq \varepsilon_{4}^{2}$, we get

$$
\begin{aligned}
& \frac{d}{d t}\left(\left\|\tilde{v}_{\tau \tau}\right\|_{0}^{2}+\left\|\tilde{\sigma}_{y y}\right\|_{0}^{2}\right)+\left\|\tilde{v}_{y y \tau}\right\|_{0}^{2}+\left\|\operatorname{div} \tilde{v}_{y y}\right\|_{0}^{2}+\left\|\tilde{\sigma}_{y y}\right\|_{0}^{2} \\
& \quad \leqq c \sqrt{\lambda}\left\|\tilde{v}_{y y y}\right\|_{0}^{2}+c(\lambda) X_{6} .
\end{aligned}
$$


Going back to old coordinates in a subdomain near the boundary, summing over all neighbourhoods, the above inequality implies (3.24).

From Lemmas 3.1 and 3.2 we have

Lemma 3.3. The following estimate holds

$$
\begin{aligned}
& \frac{d}{d t}\left(\|v\|_{1}^{2}+\left\|v_{t}\right\|_{1}^{2}+\|\omega\|_{1}^{2}+\left\|\omega_{t}\right\|_{1}^{2}+\|\sigma\|_{2}^{2}+\left\|\sigma_{t}\right\|_{0}^{2}+\left\|v_{\tau \tau}\right\|_{0}^{2}\right) \\
&+\|v\|_{2}^{2}+\left\|v_{t}\right\|_{2}^{2}+\|\omega\|_{2}^{2}+\left\|\omega_{t}\right\|_{2}^{2}+|\sigma|_{2,1}^{2}+\|\operatorname{div} v\|_{2}^{2} \\
& \leqq {\left[c(\lambda) \varepsilon_{3}+\sqrt{\lambda}\right]\|v\|_{3}^{2}+c\left(\lambda, \varepsilon_{3}\right) Y_{3}, }
\end{aligned}
$$

where $Y_{3} \equiv Y_{1}+Y_{2}$.

We shall obtain now the estimates which are crucial to conclude our argument. These estimates give a control of $\frac{d}{d t}\left(\|v\|_{2}^{2}+\|\omega\|_{2}^{2}\right)$ in terms of $\left\|v_{t}\right\|_{2}^{2}$ and $\left\|\omega_{t}\right\|_{2}^{2}$ (plus some lower order terms), and from (3.34) the final a priori estimate will follow.

Lemma 3.4. For each $0<\varepsilon_{5}<1$ the following estimate holds

$$
\begin{aligned}
& \frac{d}{d t}\left(\|\bar{A} v\|_{0}^{2}+\|\Delta \omega\|_{0}^{2}\right)+\bar{\mu}\|\nabla \bar{A} v\|_{0}^{2}+\bar{\chi}\|\nabla \Delta \omega\|_{0}^{2} \leqq \varepsilon_{5}\left(\|v\|_{3}^{2}+\|\omega\|_{3}^{2}\right) \\
& \quad+c\left[\frac{1}{\varepsilon_{5}}\left(\left\|v_{t}\right\|_{2}^{2}+\left\|\omega_{t}\right\|_{2}^{2}\right)+\|\sigma\|_{2}^{2}+\|v\|_{2}^{2}+\|\omega\|_{2}^{2}+\|f\|_{1}^{2}+g \|_{1}^{2}\right] .
\end{aligned}
$$

Proof. Writing (3.1) in the form $v_{t}+\bar{A} v=f^{*} \equiv f-p_{1} \nabla \sigma-p_{2} \nabla \omega$, and then applying the operator $\bar{A}$ to it, multiplying the result by $\bar{A} v$ and integrating over $\Omega$ we get

$$
\begin{aligned}
\frac{1}{2} \frac{d}{d t}\|\bar{A} v\|_{0}^{2} & =\int_{\Omega} \bar{A}\left(-\bar{A} v+f^{*}\right) \cdot \bar{A} v \\
& =\int_{\Omega}\left[\bar{\mu} \nabla\left(-\bar{A} v+f^{*}\right) \cdot \nabla \bar{A} v+\bar{v} \operatorname{div}\left(-\bar{A} v+f^{*}\right) \operatorname{div} \bar{A} v\right] \\
& -\int_{\partial \Omega}\left[\bar{\mu} \frac{\partial}{\partial n}\left(-\bar{A} v+f^{*}\right) \bar{A} v+\bar{v} \operatorname{div}\left(-\bar{A} v+f^{*}\right) \bar{n} \cdot \bar{A} v\right] .
\end{aligned}
$$

Therefore, by using that $v_{t}=-\bar{A} v+f^{*}$ and the immersion $H^{1,1}(\Omega) \hookrightarrow L^{1}(\partial \Omega)$, one gets

$$
\begin{aligned}
& \mid \int_{\partial \Omega} {\left[\bar{\mu} \frac{\partial}{\partial \mathrm{n}}\left(-\bar{A} v+f^{*}\right) \bar{A} v+\bar{v} \operatorname{div}\left(-\bar{A} v+f^{*}\right) \bar{n} \cdot \bar{A} v\right] \mid } \\
& \leqq c \int_{\partial \Omega}\left|\nabla v_{t}\right||\bar{A} v| \leqq c\left\|v_{t}\right\|_{2}\|v\|_{3} .
\end{aligned}
$$


Hence

$$
\begin{aligned}
& \frac{d}{d t}\|\bar{A} v\|_{0}^{2}+\bar{\mu}\|\nabla \bar{A} v\|_{0}^{2}+\bar{v}\|\operatorname{div} \bar{A} v\|_{0}^{2} \\
& \quad \leqq c\left(\left\|v_{t}\right\|_{2}\|v\|_{3}+\left\|f^{*}\right\|_{1}^{2}\right) \\
& \quad \leqq \varepsilon_{5}\|v\|_{3}^{2}+c\left(\frac{1}{\varepsilon_{5}}\left\|v_{t}\right\|_{2}^{2}+\|\sigma\|_{2}^{2}+\|\omega\|_{2}^{2}+\|f\|_{1}^{2}\right) .
\end{aligned}
$$

Repeating the above considerations for (3.3) written in the form $\omega_{t}-\bar{\chi} \Delta \omega=g^{*}$ $\equiv g-\theta_{1} p_{3} \operatorname{div} v$, one has

$$
\begin{gathered}
\frac{d}{d t}\|\Delta \omega\|_{0}^{2}+\bar{\chi}\|\nabla \Delta \omega\|_{0}^{2} \leqq c\left(\left\|\omega_{t}\right\|_{2}\|\omega\|_{3}+\left\|g^{*}\right\|_{1}^{2}\right) \\
\leqq \varepsilon_{5}\|\omega\|_{3}^{2}+c\left(\frac{1}{\varepsilon_{5}}\left\|\omega_{t}\right\|_{2}^{2}+\|v\|_{2}^{2}+\|g\|_{1}^{2}\right) .
\end{gathered}
$$

Equations (3.36) and (3.37) imply (3.35).

Finally, from Lemmas 3.3 and 3.4, for sufficiently small $\varepsilon_{5}, \lambda$, and $\varepsilon_{3}$, we have

Lemma 3.5. The following estimate holds

$$
\frac{d}{d t}\left(|v|_{2,1}^{2}+|\omega|_{2,1}^{2}+\|\sigma\|_{2}^{2}+\left\|\sigma_{t}\right\|_{0}^{2}\right)+|v|_{3,2}^{2}+|\omega|_{3,2}^{2}+|\sigma|_{2,1}^{2} \leqq c Y_{4}
$$

where

$$
\begin{aligned}
Y_{4} \equiv & |f|_{1,0}^{2}+|g|_{1,0}^{2}+\|h\|_{2}^{2}+\left\|h_{t}\right\|_{0}^{2}+\|\sigma\|_{2}^{4}+\left\|\sigma_{t}\right\|_{0}^{4}+\|v\|_{3}\|\sigma\|_{2}^{2}+\|v\|_{2}^{2}\|\sigma\|_{2}^{2} \\
& +\|\bar{u}\|_{3}\left(\|\sigma\|_{2}^{2}+\left\|\sigma_{t}\right\|_{0}^{2}\right)+\|\bar{u}\|_{2}^{2}\|\sigma\|_{2}^{2}+\left\|\bar{u}_{t}\right\|_{1}\|\sigma\|_{2}\left\|\sigma_{t}\right\|_{0}+\left|\int_{0}^{t} \int_{S_{2}} \bar{u} \cdot \bar{n}\right|^{2} .
\end{aligned}
$$

Differentiate now (3.2) with respect to $x$ and with respect to $t$. Next multiply by $\nabla \sigma_{t}$. Integrating over $\Omega$ we get

$$
\begin{aligned}
& \frac{1}{2} \frac{d}{d t}\left\|\nabla \sigma_{t}\right\|_{0}^{2} \leqq \varepsilon_{6}\left\|\sigma_{t}\right\|_{1}^{2}+\frac{c}{\varepsilon_{6}}\left(\left\|v_{t}\right\|_{2}^{2}+\left\|h_{t}\right\|_{1}^{2}\right) \\
& \quad+c\left(\|v\|_{3}+\|\bar{u}\|_{3}\right)\left\|\sigma_{t}\right\|_{1}^{2}+c\left(\left\|v_{t}\right\|_{2}+\left\|\bar{u}_{t}\right\|_{2}\right)\|\sigma\|_{2}\left\|\sigma_{t}\right\|_{1},
\end{aligned}
$$

having integrated by parts the term $\int_{\Omega}[(v+\bar{u}) \cdot \nabla] \nabla \sigma_{t} \cdot \nabla \sigma_{t}$. Moreover, the cubic terms in (3.38) and (3.39) can be estimated in this way

$$
\begin{aligned}
& \left(\|v\|_{3}+\left\|v_{t}\right\|_{2}\right)\left(\|\sigma\|_{2}^{2}+\left\|\sigma_{t}\right\|_{1}^{2}\right)+\left(\|\bar{u}\|_{3}+\left\|\bar{u}_{t}\right\|_{2}\right)\left(\|\sigma\|_{2}^{2}+\left\|\sigma_{t}\right\|_{1}^{2}\right) \\
& \quad \leqq \varepsilon_{7}\left(|v|_{3,2}^{2}+|\sigma|_{2,1}^{2}\right)+\frac{c}{\varepsilon_{7}}\left(|\sigma|_{2,1}^{4}+|\bar{u}|_{3,2}^{4}\right) .
\end{aligned}
$$

Hence from (3.38) and (3.39), by taking $\varepsilon_{6}$ and $\varepsilon_{7}$ small enough, we have at least the fundamental a priori estimate:

Lemma 3.6. The following estimate holds

$$
\frac{d}{d t}\left(|v|_{2,1}^{2}+|\omega|_{2,1}^{2}+|\sigma|_{2,1}^{2}\right)+|v|_{3,2}^{2}+|\omega|_{3,2}^{2}+|\sigma|_{2,1}^{2} \leqq \hat{c}_{0} Y_{5}
$$


where

$$
Y_{5} \equiv|f|_{1,0}^{2}+|g|_{1,0}^{2}+|h|_{2,1}^{2}+\|v\|_{2}^{2}\|\sigma\|_{2}^{2}+|\sigma|_{2,1}^{4}+|\bar{u}|_{3,2}^{4}+\left|\int_{0}^{t} \int_{S_{2}} \bar{u} \cdot \bar{n}\right|^{2} .
$$

Define now

$$
\begin{aligned}
& \varphi(t) \equiv|v(t)|_{2,1}^{2}+|\omega(t)|_{2,1}^{2}+|\sigma(t)|_{2,1}^{2} \\
& \Phi(t) \equiv|v(t)|_{3,2}^{2}+|\omega(t)|_{3,2}^{2}+|\sigma(t)|_{2,1}^{2}
\end{aligned}
$$

and

$$
\begin{gathered}
P_{1} \equiv\left|\bar{\theta}-\theta_{1}\right|_{2,1}^{2}, \\
P_{2} \equiv|\bar{u}|_{3,2}^{2}+\left|\bar{\theta}-\theta_{1}\right|_{3,2}^{2}+\left\|\bar{u}_{t t}\right\|_{0}^{2}+\left\|\bar{\theta}_{t t}\right\|_{0}^{2}+|b|_{1,0}^{2}+|r|_{1,0}^{2} .
\end{gathered}
$$

One observes at once that

$$
\Phi(t) \geqq \hat{c}_{2} \varphi(t), \quad \hat{c}_{2} \leqq 1 .
$$

Let us estimate the nonlinear terms contained in $Y_{5}$. After some long but straightforward calculations we have

Lemma 3.7. Let $p \in C^{2}, c_{v} \in C^{1}, c_{v}>0$ for $\varrho>0, \theta>0$, and (3.7) be satisfied. For simplicity one assumes that $P_{2} \leqq 1$. Then the following estimates hold

$$
\begin{gathered}
|f|_{1,0}^{2} \leqq c\left(\varphi^{2}+\varphi^{4}+\varphi \Phi+P_{2}\right), \\
|h|_{2,1}^{2} \leqq c\left(\varphi^{2}+\varphi \Phi+P_{2}\right), \\
|g|_{1,0}^{2} \leqq \hat{c}\left[\varphi^{2}+\varphi^{4}+\left(\varphi+P_{1}\right) \Phi+P_{2}\right], \\
\|v\|_{2}^{2}\|\sigma\|_{2}^{2}+|\sigma|_{2,1}^{4}+|\bar{u}|_{3,2}^{4} \leqq c\left(\varphi^{2}+P_{2}\right) .
\end{gathered}
$$

By choosing $P_{1}(t)$ so small that

$$
\hat{c}_{0} \hat{c} P_{1}(t) \leqq \frac{1}{2} \text { for each } t \in \mathbb{R}^{+},
$$

we obtain from (3.40) that

$$
\varphi_{t} \leqq-\Phi\left[1 / 2-\hat{c}_{1}\left(\varphi+\varphi^{3}\right)\right]+\hat{c}_{1}\left(P_{2}+P_{3}\right)
$$

where $P_{3}(t) \equiv\left(\int_{0}^{t} \int_{S_{2}} \bar{u} \cdot \bar{n}\right)^{2}$ and $\hat{c}_{1} \geqq 1$.

From the above estimates one can obtain the following

Lemma 3.8. Let $\bar{u} \cdot \bar{n}_{\mid \Sigma_{\infty}} \geqq 0$. Let $\partial \Omega \in C^{3}$ and let $v, \sigma$, and $\omega$ be solutions of (3.1)-(3.3) in $Q_{T}$ belonging to the classes of functions obtained in Lemma 3.6. Suppose that (3.7), (3.46) hold in $\bar{Q}_{T}$ and that

$$
\begin{gathered}
\left.\left.\varphi(0) \leqq \frac{\gamma}{2 \hat{c}_{1}}, \quad \gamma \in\right] 0, \frac{1}{2}\right], \\
P_{2}(t)+P_{3}(t)<\frac{\hat{c}_{2}}{16 \hat{c}_{1}^{2}} \gamma \quad \text { for each } t \in[0, T] .
\end{gathered}
$$


Then one has

$$
\varphi(t) \leqq \frac{\gamma}{2 \hat{c}_{1}}, \quad \forall t \in[0, T] .
$$

The terms $v_{t}(0), \omega_{t}(0), \sigma_{t}(0)$, which appear in $\varphi(0)$, have to be calculated from the Eqs. (3.1)-(3.3) evaluated at $t=0$.

Proof. Define $t^{*} \equiv \inf \left\{t \in[0, T] \mid \varphi(t)>\gamma / 2 \hat{c}_{1}\right\}$. Then $\varphi\left(t^{*}\right)=\gamma / 2 \hat{c}_{1}$, and consequently from (3.47), $\varphi_{t}\left(t^{*}\right)<0$, a contradiction. Hence $\varphi(t) \leqq \gamma / 2 \hat{c}_{1} \forall t \in[0, T]$.

On the other hand, from Sobolev's embedding theorem $H^{2}(\Omega) \hookrightarrow C^{0}(\bar{\Omega})$, one sees that there exists a constant $\hat{c}_{3}$ small enough such that, if $\varphi(t) \leqq \hat{c}_{3}$, then

$$
\varrho / 2 \leqq \sigma(t, x)+\varrho \leqq \frac{3}{2} \varrho, \quad \theta_{1} / 2 \leqq \omega(t, x)+\bar{\theta}(t, x) \leqq \frac{3}{2} \theta_{2} \quad \text { in } \quad \bar{\Omega} .
$$

Finally, we have

Lemma 3.9. There exists a constant $\hat{c}_{4}$ such that, if (3.51) holds and (for simplicity) $P_{2}(t) \leqq 1$, then

$$
\|v(t)\|_{3}^{2}+\|\omega(t)\|_{3}^{2} \leqq \hat{c}_{4}\left[\varphi(t)+\varphi^{4}(t)+P_{2}(t)\right] .
$$

Proof. By using (3.1), (3.3) one has

$$
\begin{gathered}
\|v(t)\|_{3}^{2} \leqq c\|A v(t)\|_{1}^{2} \leqq c\left[\varphi(t)+\varphi^{3}(t)+P_{2}(t)\right], \\
\|\omega(t)\|_{3}^{2} \leqq c\|\Delta \omega(t)\|_{1}^{2} \leqq c\left[\varphi(t)+\varphi^{3}(t)+P_{2}(t)\right. \\
\left.+\|v(t)\|_{3}^{2}\|v(t)\|_{2}^{2}\right] .
\end{gathered}
$$

By using the first one in the second and then adding the two estimates thus obtained, we get (3.52).

Now we are in a position to prove the global existence of a solution. In fact choose

$$
\varphi(0) \leqq \min \left(1 / 2 \hat{c}_{1}, \hat{c}_{3}\right) \equiv \hat{c}_{5}
$$

[see (3.41)], and

$$
P_{2}(t)+P_{3}(t)<\frac{1}{8} \frac{\hat{c}_{2}}{\hat{c}_{1}} \hat{c}_{5} \text { for each } t \in \mathbb{R}^{+}
$$

[see (3.43) and (3.47)]. Then we have that (3.51) holds for $\sigma_{0}(x)+\bar{\varrho}$ and $\omega_{0}(x)+\bar{\theta}(0, x)$. Moreover, from Lemma 3.9 we get that $\left\|v_{0}\right\|_{3}^{2}+\left\|\omega_{0}\right\|_{3}^{2}$ $\leqq \hat{c}_{4}\left[\hat{c}_{5}+\hat{c}_{5}^{4}+1\right] \equiv \hat{c}_{6}$. Hence from the local existence theorem we can construct a solution for $t \in\left[0, T^{*}\right]$, which satisfies (3.7) in $\bar{Q}_{T^{*}} ; T^{*}$ depends only on the data of the problem and on $\hat{c}_{5}, \hat{c}_{6}$. From Lemma 3.8 one gets

$$
\varphi(t) \leqq \hat{c}_{5}, \quad \forall t \in\left[0, T^{*}\right]
$$

and can start again from $T^{*}$, finding a solution in $\left[T^{*}, 2 T^{*}\right]$ and so on.

In this way we have proved that a global solution does exist, and satisfies (3.51) and (3.52) for each $t \in \mathbb{R}^{+}$. Therefore, we have proved 
Theorem 3.10. Let $\bar{u} \cdot \bar{n}_{\mid \partial \Omega} \geqq 0$ and

1. $\partial \Omega \in C^{3}, p \in C^{2}, p_{\varrho}\left(\bar{\varrho}, \theta_{1}\right)>0$,

$$
c_{v} \in C^{1}, c_{v}>0 \text { for } \varrho>0 \text { and } \theta>0 \text {; }
$$

2. $\bar{u} \in \Pi_{2, \infty}^{3}\left(Q_{\infty}\right) \cap \Pi_{0, \infty}^{2}\left(Q_{\infty}\right)$;

3. $\bar{\theta} \in \Pi_{2, \infty}^{3}\left(Q_{\infty}\right) \cap \Pi_{0, \infty}^{2}\left(Q_{\infty}\right) ; \theta_{1} \equiv \inf _{\Sigma_{\infty}} \bar{\theta}>0$;

4. $u_{0}-\tilde{u}(0) \in H_{0}^{1}(\Omega), \dot{v}_{0} \in H_{0}^{1}(\Omega), \varrho_{0} \in H^{2}(\Omega), \theta_{0}-\bar{\theta}(0) \in H_{0}^{1}(\Omega), \dot{\omega}_{0} \in H_{0}^{1}(\Omega)$ (see (2.21) and (2.22) for the definition of $\dot{v}_{0}$ and $\left.\dot{\omega}_{0}\right)$;

5. $r, b \in \Pi_{0, \infty}^{1}\left(Q_{\infty}\right)$;

6. in the case of an outflow: $\int_{0}^{\infty}\left(\int_{S_{2}} \bar{u} \cdot \bar{n}\right)<\infty$.

Let (3.53), (3.54) be satisfied (i.e., let $u_{0}, \bar{u}, \varrho_{0}-\bar{\varrho}, \theta_{0}-\theta_{1}, \bar{\theta}-\theta_{1}, b, r$, and $\int_{0}^{\infty}\left(\int_{S_{2}} \bar{u} \cdot \bar{n}\right)$ be small enough in suitable norms). Then there exist

$$
\begin{aligned}
(u, \theta) \in L^{\infty}\left(\mathbb{R}^{+} ; H^{3}(\Omega)\right), \quad\left(u_{t}, \theta_{t}\right) \in C_{B}^{0}\left(\mathbb{R}^{+} ; H^{1}(\Omega)\right) \cap L_{\mathrm{loc}}^{2}\left(\mathbb{R}^{+} ; H^{2}(\Omega)\right), & \\
\varrho \in C_{B}^{0}\left(\mathbb{R}^{+} ; H^{2}(\Omega)\right), & \varrho_{t} \in C_{B}^{0}\left(\mathbb{R}^{+} ; H^{1}(\Omega)\right), \\
\inf _{Q_{\infty}} \theta>0, & \inf _{Q_{\infty}} \varrho>0
\end{aligned}
$$

such that $(u, \varrho, \theta)$ is a solution of (1.1) in $Q_{\infty}$.

Remark 3.11. An analogous result can be obtained also if we merely suppose that $(\bar{u}, \bar{\theta}) \in \mathscr{S}^{2}\left(\mathbb{R}^{+} ; H^{3}(\Omega)\right), \quad\left(\bar{u}_{t}, \bar{\theta}_{t}\right) \in \mathscr{S}^{2}\left(\mathbb{R}^{+} ; H^{2}(\Omega)\right), \quad\left(\bar{u}_{t t}, \bar{\theta}_{t t}\right) \in \mathscr{S}\left(\mathbb{R}^{+} ; \quad L^{2}(\Omega)\right)$, $(b, r) \in \mathscr{S}^{2}\left(\mathbb{R}^{+} ; H^{1}(\Omega)\right),\left(b_{t}, r_{t}\right) \in \mathscr{S}^{2}\left(\mathbb{R}^{+} ; L^{2}(\Omega)\right)$ and that the norms of $\bar{u}, \bar{\theta}-\theta_{1}, b$, and $r$ in these spaces are small enough (and of course that $\varphi(0)$ and $\sup _{\mathbb{R}^{+}} P_{3}(t)$ are sufficiently small). One gets again the a priori estimates on $\varphi(t)$ (see for instance the proof of Proposition 2.2 in [14]), and consequently on $\|v(t)\|_{5 / 2}$ and $\|\omega(t)\|_{5 / 2}$ as in Lemma 3.9 (one needs to use now that

$$
\left.\|f g\|_{1 / 2} \leqq c\|f\|_{1}\|g\|_{1}, \quad\|f g\|_{1 / 2} \leqq c\|f\|_{1 / 2}\|g\|_{2}\right) .
$$

Hence the only difference is that in this situation we obtain

$$
(u, \theta) \in C_{B}^{0}\left(\mathbb{R}^{+} ; H^{5 / 2}(\Omega)\right) \cap L_{\mathrm{loc}}^{2}\left(\mathbb{R}^{+} ; H^{3}(\Omega)\right) .
$$

\section{Stability. The Case Without Outflow}

In this section we follows the methods of [31]. Suppose that $\bar{u} \cdot \bar{n} \leqq 0$ on $\Sigma_{\infty}$, and that we have two solutions $\left(v_{i}, \sigma_{i}, \omega_{i}\right), i=1,2$, of $(1.17)$ in $Q_{\infty}$ corresponding to two different initial data satisfying $\int_{\Omega} \sigma_{0}^{(1)}=\int_{\Omega} \sigma_{0}^{(2)}=0$. Suppose that both these initial data satisfy

$$
\left.\left.\varphi(0) \leqq \min \left(\frac{\gamma}{\hat{c}_{1}}, \hat{c}_{3}\right) \equiv R, \quad \gamma \in\right] 0,1 / 2\right]
$$

[see (3.41)], and

$$
P(t) \equiv P_{2}(t)+P_{3}(t)<\frac{1}{8} \frac{\hat{c}_{2}}{\hat{c}_{1}} R, \quad \forall t \in \mathbb{R}^{+}
$$


[see (3.43) and (3.47)]. When $\bar{u} \cdot \bar{n}=0$ on $\Sigma_{\infty}$, i.e., $S_{1}=\emptyset$, from Lemma 3.8 we know that the corresponding solutions satisfy

$$
\varphi_{i}(t) \leqq R, \quad i=1,2,
$$

for each $t \in \mathbb{R}^{+}$. If $S_{1} \neq \emptyset$, assume that (4.3) is satisfied. From (3.47) we easily get (again for $S_{1}=\emptyset$ )

$$
\frac{1}{8} \int_{0}^{t} \Phi_{i}(s) d s \leqq R+\hat{c}_{1} \int_{0}^{t} P(s) d s \leqq R\left(1+\frac{\hat{c}_{2}}{8} t\right), \quad i=1,2 .
$$

If $S_{1} \neq \emptyset$, assume that (4.4) holds (see, however, Theorem 4.3). By choosing $\gamma$ small enough, we can make $R$ as small as we need. From now on we shall assume that $R \leqq 1$.

Set now $q \equiv v_{1}-v_{2}, \eta \equiv \sigma_{1}-\sigma_{2}, \beta \equiv \omega_{1}-\omega_{2}$. We want to prove that all the solutions of (1.17) are asymptotically equivalent. More precisely, we shall prove that

$$
\begin{aligned}
& \|q(t)\|_{0}^{2}+\|\eta(t)\|_{0}^{2}+\|\beta(t)\|_{0}^{2} \\
& \quad \leqq c\left(\|q(0)\|_{0}^{2}+\|\eta(0)\|_{0}^{2}+\|\beta(0)\|_{0}^{2}\right) \exp (-\varepsilon t),
\end{aligned}
$$

$\varepsilon>0, t \in \mathbb{R}^{+}$. Now we write the equations for $q, \eta$, and $\beta$.

$$
\begin{gathered}
q_{t}+\bar{A} q+p_{1} \nabla \eta+p_{2} \nabla \beta=f_{1}-f_{2} \text { in } Q_{\infty}, \\
q=0 \quad \text { on } \Sigma_{\infty}, \\
q(0)=q_{0} \equiv v_{0}^{(1)}-v_{0}^{(2)} \text { in } \Omega ; \\
\eta_{t}+\varrho \operatorname{div} q=h_{1}-h_{2}-\left(v_{1}+\bar{u}\right) \cdot \nabla \eta-q \nabla \sigma_{2} \text { in } Q_{\infty}, \\
\eta=0 \quad \text { on }] 0, \infty\left[\times S_{1},\right. \\
\eta(0)=\eta_{0} \equiv \sigma_{0}^{(1)}-\sigma_{0}^{(2)} \quad \text { in } \Omega ; \\
\beta_{t}-\bar{\chi} \Delta \beta+\theta_{1} p_{3} \operatorname{div} q=g_{1}-g_{2} \text { in } Q_{\infty}, \\
\beta=0 \quad \text { on } \Sigma_{\infty}, \\
\beta(0)=\beta_{0} \equiv \omega_{0}^{(1)}-\omega_{0}^{(2)} \text { in } \Omega,
\end{gathered}
$$

where

$$
\begin{aligned}
f_{1}^{1}-f_{2}^{1}= & {\left[p_{1}-\frac{p_{\varrho}\left(\sigma_{1}+\bar{\varrho}, \omega_{1}+\bar{\theta}\right)}{\sigma_{1}+\bar{\varrho}}\right] \nabla \eta } \\
& +\left[\frac{p_{\varrho}\left(\sigma_{2}+\bar{\varrho}, \omega_{2}+\bar{\theta}\right)}{\sigma_{2}+\bar{\varrho}}-\frac{p_{\varrho}\left(\sigma_{1}+\bar{\varrho}, \omega_{1}+\bar{\theta}\right)}{\sigma_{1}+\bar{\varrho}}\right] \nabla \sigma_{2}, \\
f_{1}^{2}-f_{2}^{2}= & {\left[p_{2}-\frac{p_{\theta}\left(\sigma_{1}+\bar{\varrho}, \omega_{1}+\bar{\theta}\right)}{\sigma_{1}+\bar{\varrho}}\right] \nabla \beta } \\
& +\left[\frac{p_{\theta}\left(\sigma_{2}+\bar{\varrho}, \omega_{2}+\bar{\theta}\right)}{\sigma_{2}+\bar{\varrho}}-\frac{p_{\theta}\left(\sigma_{1}+\bar{\varrho}, \omega_{1}+\bar{\theta}\right)}{\sigma_{1}+\bar{\varrho}}\right] \nabla\left(\omega_{2}+\bar{\theta}\right), \\
f_{1}^{3}-f_{2}^{3}= & -\left(v_{1}+\bar{u}\right) \cdot \nabla q-q \cdot \nabla\left(v_{2}+\bar{u}\right) \\
& +\frac{\sigma_{2}}{\sigma_{2}+\bar{\varrho}} \bar{A} q+\frac{\eta}{\left(\sigma_{1}+\bar{\varrho}\right)\left(\sigma_{2}+\bar{\varrho}\right)} A v_{1}+\frac{\eta}{\left(\sigma_{1}+\bar{\varrho}\right)\left(\sigma_{2}+\bar{\varrho}\right)} A \bar{u},
\end{aligned}
$$


and

$$
h_{1}-h_{2}=-\eta \operatorname{div} v_{1}-\sigma_{2} \operatorname{div} q-\eta \operatorname{div} \bar{u} .
$$

We can write also

$$
h_{1}-h_{2}-\left(v_{1}+\bar{u}\right) \cdot \nabla \eta-q \cdot \nabla \sigma_{2}=-\operatorname{div}\left[\sigma_{2} q+\left(v_{1}+\bar{u}\right) \eta\right]
$$

At last we have

$$
\begin{aligned}
& g_{1}^{1}-g_{2}^{1}=\left[\theta_{1} \frac{p_{\theta}\left(\bar{\varrho}, \theta_{1}\right)}{\varrho \varrho c_{v}\left(\bar{\varrho}, \theta_{1}\right)}-\frac{\left(\omega_{1}+\bar{\theta}\right) p_{\theta}\left(\sigma_{1}+\bar{\varrho}, \omega_{1}+\bar{\theta}\right)}{\left(\sigma_{1}+\bar{\varrho}\right) c_{v}\left(\sigma_{1}+\bar{\varrho}, \omega_{1}+\bar{\theta}\right)}\right] \operatorname{div} q \\
& +\left[\frac{\left(\omega_{2}+\bar{\theta}\right) p_{\theta}\left(\sigma_{2}+\bar{\varrho}, \omega_{2}+\bar{\theta}\right)}{\left(\sigma_{2}+\bar{\varrho}\right) c_{v}\left(\sigma_{2}+\bar{\varrho}, \omega_{2}+\bar{\theta}\right)}\right. \\
& \left.-\frac{\left(\omega_{1}+\bar{\theta}\right) p_{\theta}\left(\sigma_{1}+\bar{\varrho}, \omega_{1}+\bar{\theta}\right)}{\left(\sigma_{1}+\bar{\varrho}\right) c_{v}\left(\sigma_{1}+\bar{\varrho}, \omega_{1}+\bar{\theta}\right)}\right] \operatorname{div} v_{2} \\
& -\left[\bar{\chi}-\frac{\chi}{\left(\sigma_{2}+\bar{\varrho}\right) c_{v}\left(\sigma_{2}+\bar{\varrho}, \omega_{2}+\bar{\theta}\right)}\right] \Delta \beta \\
& -\chi\left[\frac{1}{\left(\sigma_{1}+\varrho\right) c_{v}\left(\sigma_{1}+\bar{\varrho}, \omega_{1}+\bar{\theta}\right)}-\frac{1}{\left(\sigma_{2}+\varrho\right) c_{v}\left(\sigma_{2}+\varrho, \omega_{2}+\bar{\theta}\right)}\right] \Delta \omega_{1} \text {, } \\
& g_{1}^{2}-g_{2}^{2}=\chi\left[\frac{1}{\left(\sigma_{1}+\bar{\varrho}\right) c_{v}\left(\sigma_{1}+\bar{\varrho}, \omega_{1}+\bar{\theta}\right)}-\frac{1}{\left(\sigma_{2}+\varrho\right) c_{v}\left(\sigma_{2}+\bar{\varrho}, \omega_{2}+\bar{\theta}\right)}\right] \Delta \bar{\theta} \\
& -\left[\frac{\left(\omega_{1}+\bar{\theta}\right) p_{\theta}\left(\sigma_{1}+\bar{\varrho}, \omega_{1}+\bar{\theta}\right)}{\left(\sigma_{1}+\varrho\right) c_{v}\left(\sigma_{1}+\varrho, \omega_{1}+\bar{\theta}\right)}\right.
\end{aligned}
$$

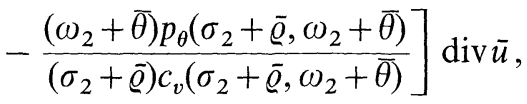

$$
\begin{aligned}
& g_{1}^{3}-g_{2}^{3}=-q \cdot \nabla\left(\omega_{1}+\bar{\theta}\right)-\left(v_{2}+\bar{u}\right) \cdot \nabla \beta+\frac{1}{\left(\sigma_{1}+\bar{\varrho}\right) c_{v}\left(\sigma_{1}+\bar{\varrho}, \omega_{1}+\bar{\theta}\right)} \\
& \text {. }\left\{\frac{\mu}{2}\left(D_{i} q^{k}+D_{k} q^{i}\right)\left[D_{k}\left(v_{1}+v_{2}+2 \bar{u}\right)^{i}+D_{i}\left(v_{1}+v_{2}+2 \bar{u}\right)^{k}\right]\right. \\
& \left.+(v-\mu) \operatorname{div} q \operatorname{div}\left(v_{1}+v_{2}+2 \bar{u}\right)\right\} \\
& +\left[\frac{1}{\left(\sigma_{1}+\bar{\varrho}\right) c_{v}\left(\sigma_{1}+\bar{\varrho}, \omega_{1}+\bar{\theta}\right)}-\frac{1}{\left(\sigma_{2}+\bar{\varrho}\right) c_{v}\left(\sigma_{2}+\bar{\varrho}, \omega_{2}+\bar{\theta}\right)}\right] \\
& \text {. }\left\{\frac{\mu}{2} \sum_{i, k}\left[D_{k}\left(v_{2}+\tilde{u}\right)^{i}+D_{i}\left(v_{2}+\bar{u}\right)^{k}\right]^{2}+(v-\mu)\left[\operatorname{div}\left(v_{2}+\bar{u}\right)\right]^{2}\right\} \\
& +r\left[\frac{1}{c_{v}\left(\sigma_{1}+\bar{\varrho}, \omega_{1}+\bar{\theta}\right)}-\frac{1}{c_{v}\left(\sigma_{2}+\bar{\varrho}, \omega_{2}+\bar{\theta}\right)}\right] \text {. }
\end{aligned}
$$


From (4.6)-(4.8), by proceeding as in Lemma 3.1 (see also Lemma 5.1 in [31]), we have for each $0<\varepsilon<1$,

$$
\begin{aligned}
& \frac{1}{2} \frac{d}{d t}\|q\|_{0}^{2}+\frac{1}{2} \frac{p_{1}}{\bar{\varrho}} \frac{d}{d t}\|\eta\|_{0}^{2}+\frac{p_{2}}{2 p_{3} \theta_{1}} \frac{d}{d t}\|\beta\|_{0}^{2} \\
& \quad+\frac{\bar{\mu}}{2}\|\nabla q\|_{0}^{2}+\bar{v}\|\operatorname{div} q\|_{0}^{2}+\frac{\bar{\chi} p_{2}}{2 p_{3} \theta_{1}}\|\nabla \beta\|_{0}^{2} \\
& \leqq c\left(\left\|f_{1}-f_{2}\right\|_{-1}^{2}+\left\|g_{1}-g_{2}\right\|_{-1}^{2}+\frac{1}{\varepsilon}\left\|h_{1}-h_{2}\right\|_{0}^{2}\right)+\varepsilon\|\eta\|_{0}^{2} \\
& \quad+\frac{c}{\varepsilon} R\|q\|_{1}^{2}+\frac{c}{\varepsilon}\left(\left\|v_{1}\right\|_{3}^{2}+\|\bar{u}\|_{3}^{2}\right)\|\eta\|_{0}^{2} .
\end{aligned}
$$

Moreover, after some straightforward calculations, one gets

Lemma 4.1. Let $\bar{S}_{1} \equiv\left\|\bar{\theta}-\theta_{1}\right\|_{2}^{2} \leqq 1$ (for simplicity), $\bar{S}_{2} \equiv\|\bar{u}\|_{3}^{2}+\|\bar{u}\|_{2}^{4}+\left\|\bar{\theta}-\theta_{1}\right\|_{3}^{2}$ $+\|r\|_{1}^{2}, p \in C^{2}, c_{v} \in C^{1}$ and (3.7) be satisfied. Then the following estimates are valid

$$
\begin{gathered}
\left\|f_{1}-f_{2}\right\|_{-1}^{2} \leqq c\left(R+\bar{S}_{1}\right)\left(\|\eta\|_{0}^{2}+\|\beta\|_{0}^{2}\right)+c R\|q\|_{1}^{2} \\
+c\left(\left\|v_{1}\right\|_{3}^{2}+\bar{S}_{2}\right)\left(\|\eta\|_{0}^{2}+\|q\|_{0}^{2}\right), \\
\left\|h_{1}-h_{2}\right\|_{0}^{2} \leqq c R\|q\|_{1}^{2}+c\left(\left\|v_{1}\right\|_{3}^{2}+\bar{S}_{2}\right)\|\eta\|_{0}^{2}, \\
\left\|g_{1}-g_{2}\right\|_{-1}^{2} \leqq \\
c\left(R+\bar{S}_{1}\right)\left(\|\beta\|_{1}^{2}+\|q\|_{1}^{2}\right) \\
+c\left(\left\|\omega_{1}\right\|_{3}^{2}+R+\bar{S}_{2}\right)\left(\|\eta\|_{0}^{2}+\|q\|_{0}^{2}+\|\beta\|_{0}^{2}\right) .
\end{gathered}
$$

Using (4.14)-(4.16) in (4.13) one obtains

$$
\begin{aligned}
\frac{1}{2} \frac{d}{d t} & \|q\|_{0}^{2}+\frac{1}{2} \frac{p_{1}}{\bar{\varrho}} \frac{d}{d t}\|\eta\|_{0}^{2}+\frac{p_{2}}{2 p_{3} \theta_{1}} \frac{d}{d t}\|\beta\|_{0}^{2} \\
& +\frac{\bar{\mu}}{2}\|\nabla q\|_{0}^{2}+\bar{v}\|\operatorname{div} q\|_{0}^{2}+\frac{\bar{\chi} p_{2}}{2 p_{3} \theta_{1}}\|\nabla \beta\|_{0}^{2} \\
\leqq & c\left(R+\bar{S}_{1}\right)\left(\|\eta\|_{0}^{2}+\|\beta\|_{1}^{2}+\|q\|_{1}^{2}\right)+\varepsilon\|\eta\|_{0}^{2}+\frac{c}{\varepsilon} R\|q\|_{1}^{2} \\
& +\frac{c}{\varepsilon}\left(\left\|v_{1}\right\|_{3}^{2}+\bar{S}_{2}\right)\left(\|\eta\|_{0}^{2}+\|q\|_{0}^{2}\right) \\
& +c\left(\left\|\omega_{1}\right\|_{3}^{2}+\bar{S}_{2}\right)\left(\|\eta\|_{0}^{2}+\|q\|_{0}^{2}+\|\beta\|_{0}^{2}\right) .
\end{aligned}
$$

Now we want to estimate $\|\eta\|_{0}^{2}$. We can repeat essentially the considerations of [31]. Let $z$ be the solution of the Stokes' problem

$$
\begin{aligned}
\Delta z-\nabla \pi=0 & \text { in } \quad Q_{\infty}, \\
\operatorname{div} z=\eta & \text { in } \quad Q_{\infty}, \\
z=0 & \text { on } \quad \Sigma_{\infty},
\end{aligned}
$$


which exists since $\int_{\Omega} \eta=\int_{\Omega} \eta_{0}-\int_{0}^{t} \int_{\partial \Omega}\left[\eta \bar{u} \cdot \bar{n}+\left(\sigma_{2}+\bar{\varrho}\right) q \cdot \bar{n}\right]=0$ for each $t \in \mathbb{R}^{+}$, because $\bar{u} \cdot \bar{n}=0$ on $\partial \Omega \backslash S_{1}$ and $\eta=0$ on $S_{1}$. Therefore, we have

$$
\|z\|_{1}^{2} \leqq c\|\eta\|_{0}^{2} \text {. }
$$

Multiplying (4.6) ${ }_{1}$ by $z$ and integrating over $\Omega$, from (4.19) one has

$$
\begin{aligned}
p_{1}\|\eta\|_{0}^{2} & =\int_{\Omega}\left[q_{t}+\bar{A} q+p_{2} \nabla \beta-\left(f_{1}-f_{2}\right)\right] \cdot z \\
& \leqq \int_{\Omega} q_{t} \cdot z+c\left(\|\bar{A} q\|_{-1}+\|\nabla \beta\|_{-1}+\left\|f_{1}-f_{2}\right\|_{-1}\right)\|\eta\|_{0} \\
& \leqq \int_{\Omega} q_{t} \cdot z+c\left(\|q\|_{1}^{2}+\|\beta\|_{0}^{2}+\left\|f_{1}-f_{2}\right\|_{-1}^{2}\right)+\frac{p_{1}}{2}\|\eta\|_{0}^{2} .
\end{aligned}
$$

On the other hand

$$
\int_{\Omega} q_{t} \cdot z=\frac{d}{d t} \int_{\Omega} q \cdot z-\int_{\Omega} q \cdot z_{t} .
$$

By taking the time derivative of (4.18) we see that $z_{t}$ is the solution of

$$
\begin{aligned}
\Delta z_{t}-\nabla \pi_{t}=0 & \text { in } \quad Q_{\infty}, \\
\operatorname{div} z_{t}=\eta_{t} & \text { in } \quad Q_{\infty}, \\
z_{t}=0 & \text { on } \quad \Sigma_{\infty},
\end{aligned}
$$

and from (4.11)

$$
\eta_{t}=-\operatorname{div} W
$$

where

$$
W \equiv\left(\sigma_{2}+\varrho \bar{\varrho}\right) q+\left(v_{1}+\bar{u}\right) \eta
$$

Let $(\mathscr{V}, \mathscr{P})$ be a solution of the Stokes' problem

$$
\begin{array}{rlc}
\Delta \mathscr{V}-\nabla \mathscr{P}=q & \text { in } \quad Q_{\infty}, \\
\operatorname{div} \mathscr{V}=0 & \text { in } \quad Q_{\infty}, \\
\mathscr{V}=0 & \text { on } \quad \Sigma_{\infty},
\end{array}
$$

which satisfies

$$
\|\mathscr{V}\|_{2}+\|\nabla \mathscr{P}\|_{0} \leqq c\|q\|_{0} .
$$

Hence, by recalling again that $W \cdot \bar{n}_{\mid \partial \Omega}=0$ since $\bar{u} \cdot \bar{n}=0$ on $\partial \Omega \backslash S_{1}$ and $\eta=0$ on $S_{1}$, one gets

$$
\begin{aligned}
\int_{\Omega} q \cdot z_{t} & =\int_{\Omega}(\Delta \mathscr{V}-\nabla \mathscr{P}) \cdot z_{t}=\int_{\Omega} \mathscr{V} \cdot \Delta z_{t}+\mathscr{P} \operatorname{div} z_{t} \\
& =\int_{\Omega} \mathscr{V} \cdot \nabla \pi_{t}-\int_{\Omega} \mathscr{P} \operatorname{div} W=\int_{\Omega} \nabla \mathscr{P} \cdot W
\end{aligned}
$$

and estimating $W$,

$$
\left|\int_{\Omega} q \cdot z_{t}\right| \leqq c\|q\|_{0}^{2}+c\left(R+\bar{S}_{2}\right)\|\eta\|_{0}^{2} .
$$


Multiplying (4.20) by $\delta, 0<\delta<1$, and recalling the estimate for $\left\|f_{1}-f_{2}\right\|_{-1}^{2}$, we obtain

$$
\begin{aligned}
& \delta \frac{p_{1}}{2}\|\eta\|_{0}^{2}-\delta \frac{d}{d t} \int_{\Omega} q \cdot z \\
& \quad \leqq c \delta\left(\|q\|_{1}^{2}+\|\beta\|_{0}^{2}\right)+c \delta\left(R+\bar{S}_{2}+\left\|v_{1}\right\|_{3}^{2}\right)\left(\|\eta\|_{0}^{2}+\|q\|_{0}^{2}+\|\beta\|_{0}^{2}\right) .
\end{aligned}
$$

By choosing $\varepsilon=\delta p_{1} / 4$, from (4.17) and (4.24) we have

$$
\begin{aligned}
\frac{1}{2} \frac{d}{d t} & \|q\|_{0}^{2}+\frac{1}{2} \frac{p_{1}}{\varrho} \frac{d}{d t}\|\eta\|_{0}^{2}+\frac{p_{2}}{2 p_{3} \theta_{1}} \frac{d}{d t}\|\beta\|_{0}^{2}+\frac{\bar{\mu}}{2}\|\nabla q\|_{0}^{2}+\bar{v}\|\operatorname{div} q\|_{0}^{2} \\
& +\frac{\bar{\chi} p_{2}}{2 p_{3} \theta_{1}}\|\nabla \beta\|_{0}^{2}+\delta \frac{p_{1}}{4}\|\eta\|_{0}^{2}-\delta \frac{d}{d t} \int_{\Omega} q \cdot z \\
\leqq & c\left(R+\bar{S}_{1}\right)\left(\|\eta\|_{0}^{2}+\|q\|_{1}^{2}+\|\beta\|_{1}^{2}\right)+\frac{c}{\delta} R\|q\|_{1}^{2}+c \delta\left(\|q\|_{1}^{2}+\|\beta\|_{0}^{2}\right) \\
& +c\left(\delta+\frac{1}{\delta}\right)\left(\bar{S}_{2}+\left\|v_{1}\right\|_{3}^{2}+\left\|\omega_{1}\right\|_{3}^{2}\right)\left(\|\eta\|_{0}^{2}+\|q\|_{0}^{2}+\|\beta\|_{0}^{2}\right) .
\end{aligned}
$$

Now we integrate (4.25) with respect to $t$. Since [because of (4.19)]

$$
\delta\left|\int_{\Omega} q \cdot z\right| \leqq \bar{c}_{1} \delta\left(\|q\|_{0}^{2}+\|\eta\|_{0}^{2}\right)
$$

we get

$$
\begin{aligned}
\left(\frac{1}{2}-\right. & \left.\bar{c}_{1} \delta\right)\|q\|_{0}^{2}+\left(\frac{1}{2} \frac{p_{1}}{\bar{\varrho}}-\bar{c}_{1} \delta\right)\|\eta\|_{0}^{2}+\frac{p_{2}}{2 p_{3} \theta_{1}}\|\beta\|_{0}^{2} \\
& +\frac{\bar{\mu}}{2} \int_{0}^{t}\|\nabla \mathrm{q}\|_{0}^{2}+\bar{v} \int_{0}^{t}\|\operatorname{div} q\|_{0}^{2}+\frac{\bar{\chi} p_{2}}{2 p_{3} \theta_{1}} \int_{0}^{t}\|\nabla \beta\|_{0}^{2}+\delta \frac{p_{1}}{4} \int_{0}^{t}\|\eta\|_{0}^{2} \\
\leqq & \bar{c}_{2}\left(\left\|\eta_{0}\right\|_{0}^{2}+\left\|q_{0}\right\|_{0}^{2}+\left\|\beta_{0}\right\|_{0}^{2}\right) \\
& +\bar{c}_{2}\left(R+\bar{S}_{1}\right) \int_{0}^{t}\left(\|\eta\|_{0}^{2}+\|\nabla \beta\|_{0}^{2}+\|\nabla q\|_{0}^{2}\right) \\
& +\frac{\bar{c}_{2}}{\delta} R \int_{0}^{t}\|\nabla q\|_{0}^{2}+\bar{c}_{2} \delta \int_{0}^{t}\left(\|\nabla q\|_{0}^{2}+\|\nabla \beta\|_{0}^{2}\right) \\
& +\bar{c}_{2}\left(\delta+\frac{1}{\delta}\right) \int_{0}^{t}\left(\bar{S}_{2}+\left\|v_{1}\right\|_{3}^{2}+\left\|\omega_{1}\right\|_{3}^{2}\right)\left(\|\eta\|_{0}^{2}+\|q\|_{0}^{2}+\|\beta\|_{0}^{2}\right) .
\end{aligned}
$$

Assuming that

$$
\delta \leqq \min \left\{\frac{1}{4 \bar{c}_{1}}, \frac{p_{1}}{4 \varrho \bar{c}_{1}}, \frac{\bar{\mu}}{4 \bar{c}_{2}}, \frac{\bar{\chi} p_{2}}{4 p_{3} \theta_{1} \bar{c}_{2}}, 1\right\}
$$

and

$$
\bar{S}_{1}+R \leqq \min \left\{\frac{\delta p_{1}}{8 \bar{c}_{2}}, \frac{\bar{\chi} p_{2}}{8 \bar{c}_{2} p_{3} \theta_{1}}, \frac{\delta \bar{\mu}}{8 \bar{c}_{2}(1+\delta)}, 1\right\}
$$


[see (4.1) and Lemma 4.1], we have

$$
\begin{aligned}
& \|q\|_{0}^{2}+\|\eta\|_{0}^{2}+\|\beta\|_{0}^{2}+\int_{0}^{t}\left(\|\nabla q\|_{0}^{2}+\|\operatorname{div} q\|_{0}^{2}+\|\nabla \beta\|_{0}^{2}+\|\eta\|_{0}^{2}\right) \\
& \leqq \bar{c}_{3}\left(\left\|q_{0}\right\|_{0}^{2}+\left\|\eta_{0}\right\|_{0}^{2}+\left\|\beta_{0}\right\|_{0}^{2}\right) \\
& \quad+\bar{c}_{3} \int_{0}^{t}\left(\left\|v_{1}\right\|_{3}^{2}+\left\|\omega_{1}\right\|_{3}^{2}+\bar{S}_{2}\right)\left(\|q\|_{0}^{2}+\|\eta\|_{0}^{2}+\|\beta\|_{0}^{2}\right)
\end{aligned}
$$

Putting

$$
\psi(t) \equiv\|q(t)\|_{0}^{2}+\|\eta(t)\|_{0}^{2}+\|\beta(t)\|_{0}^{2}+\int_{0}^{t}\left(\|\nabla q\|_{0}^{2}+\|\operatorname{div} q\|_{0}^{2}+\|\nabla \beta\|_{0}^{2}+\|\eta\|_{0}^{2}\right)
$$

and

$$
\zeta(t) \equiv\left\|v_{1}(t)\right\|_{3}^{2}+\left\|\omega_{1}(t)\right\|_{3}^{2}+\bar{S}_{2}(t),
$$

(4.29) can be written as

$$
\psi(t) \leqq \bar{c}_{3}\left[\psi(0)+\int_{0}^{t} \zeta(s) \psi(s) d s\right]
$$

hence by Gronwall's lemma one has

$$
\begin{aligned}
\psi(t) & \leqq \bar{c}_{3} \psi(0) \exp \left[\bar{c}_{3} \int_{0}^{t} \zeta(s) d s\right] \\
& \leqq \bar{c}_{3} \psi(0) \exp \left[8 \bar{c}_{3} R\left(1+\frac{\hat{c}_{2}}{8} t\right)+\bar{c}_{3} \int_{0}^{t} \bar{S}_{2}\right]
\end{aligned}
$$

By (4.2) we have that

$$
\int_{0}^{t} \bar{S}_{2} \leqq c R t
$$

Introducing

$$
\begin{gathered}
\tilde{q}(t, x) \equiv \exp (\alpha t) q(t, x), \quad \tilde{\eta}(t, x) \equiv \exp (\alpha t) \eta(t, x), \\
\tilde{\beta}(t, x) \equiv \exp (\alpha t) \beta(t, x),
\end{gathered}
$$

and repeating the considerations from [31] we get, for a sufficiently small $\alpha$,

$$
\begin{aligned}
\|q(t)\|_{0}^{2} & +\|\eta(t)\|_{0}^{2}+\|\beta(t)\|_{0}^{2} \\
& +\int_{0}^{t} \exp [-2 \alpha(t-s)]\left(\|\nabla q(s)\|_{0}^{2}+\|\nabla \beta(s)\|_{0}^{2}+\|\eta(s)\|_{0}^{2}\right) d s \\
\leqq & c \exp (c R)\left(\left\|q_{0}\right\|_{0}^{2}+\left\|\eta_{0}\right\|_{0}^{2}+\left\|\beta_{0}\right\|_{0}^{2}\right) \exp \left[-\left(2 \alpha-\bar{c}_{4} R\right) t\right]
\end{aligned}
$$

for each $t \in \mathbb{R}^{+}$.

Hence we have proved:

Theorem 4.2. Let the assumptions 1-5 of Theorem 3.10 be satisfied, and $\bar{u} \cdot \bar{n}_{\mid \Sigma_{\infty}}=0$. Assume also that (4.1) and (4.2) are satisfied for $\gamma$ small enough in such a way that 
(4.28) holds and

$$
2 \alpha-\bar{c}_{4} R>0
$$

(see (4.35)). Let $\left(u_{i}^{*}, \varrho_{i}^{*}, \theta_{i}^{*}\right)$ be the solutions of (1.1) in $Q_{\infty}$ corresponding to the initial data $\left(u_{0}^{(i)}, \varrho_{0}^{(i)}, \theta_{0}^{(i)}\right)$, satisfying $\int_{\Omega} \varrho_{0}^{(i)}=\varrho \operatorname{vol}(\Omega), i=1,2$. Then the difference $(q, \eta, \beta)$ between $\left(u_{1}^{*}, \varrho_{1}^{*}, \theta_{1}^{*}\right)$ and $\left(u_{2}^{*}, \varrho_{2}^{*}, \theta_{2}^{*}\right)$ satisfies $(4.35)$ and tends to zero as $t \rightarrow \infty$.

Theorem 4.3. Let $\left(u_{1}^{*}, \varrho_{1}^{*}, \theta_{1}^{*}\right)$ and $\left(u_{2}^{*}, \varrho_{2}^{*}, \theta_{2}^{*}\right)$ be two solutions of $(1.1),(1.5)$ such that $\int_{\Omega} \varrho_{0}^{(1)}=\int_{\Omega} \varrho_{0}^{(2)}=\bar{\varrho} \operatorname{vol}(\Omega)$ and

$$
\begin{gathered}
\left\|u_{i}^{*}(t)-\bar{u}(t)\right\|_{2}^{2}+\left\|\varrho_{i}^{*}(t)-\bar{\varrho}\right\|_{2}^{2}+\left\|\theta_{i}^{*}(t)-\bar{\theta}(t)\right\|_{2}^{2} \leqq N_{1}, \quad \forall t \in \mathbb{R}^{+}, \quad i=1,2 \\
\int_{0}^{t}\left(\left\|u_{1}^{*}(s)-\bar{u}(s)\right\|_{3}^{2}+\left\|\theta_{1}^{*}(s)-\bar{\theta}(s)\right\|_{3}^{2}\right) d s \leqq N_{2}+N_{3} t, \quad \forall t \in \mathbb{R}^{+} .
\end{gathered}
$$

Suppose that

$$
\begin{gathered}
\left\|\bar{\theta}(t)-\theta_{1}\right\|_{2}^{2} \leqq N_{4}, \quad \forall t \in \mathbb{R}^{+}, \quad\left(\theta_{1}=\inf _{\Sigma_{\infty}} \bar{\theta}\right) \\
\int_{0}^{t}\left(\|\bar{u}(s)\|_{3}^{2}+\|\bar{u}(s)\|_{2}^{4}+\left\|\bar{\theta}(s)-\theta_{1}\right\|_{3}^{2}+\|r(s)\|_{1}^{2}\right) d s \leqq N_{5}+N_{6} t, \quad \forall t \in \mathbb{R}^{+},
\end{gathered}
$$

and that $\bar{u} \cdot \bar{n}_{\mid \Sigma_{\infty}} \leqq 0$. If $N_{1}, N_{3}, N_{4}$, and $N_{6}$ are small enough, then the difference $(q, \eta, \beta)$ between $\left(u_{1}^{*}, \varrho_{1}^{*}, \theta_{1}^{*}\right)$ and $\left(u_{2}^{*}, \varrho_{2}^{*}, \theta_{2}^{*}\right)$ goes to zero in $L^{2}(\Omega)$ exponentially as $t \rightarrow \infty$.

Remark 4.4. Conditions (4.39) and (4.40) are satisfied if $\bar{u}, \bar{\theta}$, and $r$ are as in Remark 3.11.

\section{Periodic and Stationary Solutions. The Case Without Inflow and Outflow}

It is well known that from the global existence theorem and from stability it follows that there exists a $T$-periodic solution under the action of $T$-periodic external forces (and heat sources) and boundary data (see, for instance, Serrin $[24,25]$ ).

Let us explain briefly this argument: one constructs a global solution $U^{*}$ by starting from some initial data, and then considers $U_{n}(x) \equiv U^{*}(n T, x)$. By the stability result, and using the periodicity of the data, one gets that $U_{n} \rightarrow U_{\infty}$ strongly in $L^{2}(\Omega)$ (and, by compactness, in some stronger topology). Finally, one proves that the solution having $U_{\infty}$ as initial data is a $T$-periodic solution.

We are now in a position to apply this method for showing the existence of a $T$ periodic solution $(u, \varrho, \theta)$ of $(1.1)_{1}-(1.1)_{3},(1.1)_{5},(1.1)_{7}$ with $\int_{\Omega} \varrho=\bar{\varrho} \operatorname{vol}(\Omega), \bar{\varrho}$ a given
positive constant. We have only to verify that:

(i) given $b, r, \bar{u}, \bar{\theta}$ satisfying (4.2), $\bar{u} \cdot \bar{n}=0$ on $\partial \Omega$, it is possible to find initial data $\left(v_{0}, \sigma_{0}, \omega_{0}\right)$ which satisfy the assumption 4 of Theorem 3.10 (in particular the compatibility conditions) and such that (4.1) is satisfied; (ii) the limit of $\left(v^{*}(n T)\right.$, $\left.\sigma^{*}(n T), \omega^{*}(n T)\right)$, say $\left(v_{\infty}, \sigma_{\infty}, \omega_{\infty}\right)$, satisfies the assumption 4 of Theorem 3.10 and (4.1). 
To verify (i), one can proceed in several ways. For instance, extend $\bar{u}$ and $\bar{\theta}$ in $[-T, \infty[$ by periodicity, and choose an extension $\tilde{b}$ and $\tilde{r}$ of $b$ and $r$ in $[-T, \infty[$, so that

$$
\begin{aligned}
& \tilde{b}(-T)=\frac{1}{\bar{\varrho}} \nabla[p(\bar{\varrho}, \bar{\theta}(0))]+\bar{A} \bar{u}(0)+\bar{u}_{t}(0)+\bar{u}(0) \cdot \nabla \bar{u}(0) \text { in } \Omega, \\
& \tilde{r}(-T)= \frac{1}{\bar{\varrho}} \bar{\theta}(0) p_{\theta}(\bar{\varrho}, \bar{\theta}(0)) \operatorname{div} \bar{u}(0)-\frac{\chi}{\bar{\varrho}} \Delta \bar{\theta}(0) \\
&-\frac{\mu}{2 \varrho} \sum_{i, k}\left(D_{k} \bar{u}^{i}(0)+D_{i} \bar{u}^{k}(0)\right)^{2} \\
&-\frac{1}{\bar{\varrho}}(v-\mu)(\operatorname{div} \bar{u}(0))^{2}+c_{v}(\bar{\varrho}, \bar{\theta}(0)) \\
& \cdot\left[\bar{\theta}_{t}(0)+\bar{u}(0) \cdot \nabla \bar{\theta}(0)\right] \text { in } \Omega .
\end{aligned}
$$

One can proceed in such a way that the function $\widetilde{\mathscr{P}}_{2}(t)$ [defined as in (3.43)] satisfies

$$
\mathscr{P}_{1}(t)+\widetilde{\mathscr{P}}_{2}(t) \leqq \bar{c}_{5} R, \quad \forall t \in[-T, \infty[.
$$

Choose now as initial data in $t_{0}=-T$ the functions $v_{0}=0, \sigma_{0}=0, \omega_{0}=0$. By (5.1), (5.2) we obtain $\varphi(-T)=0$. Hence by applying Theorem 3.10 we get a global solution $(v, \sigma, \omega)$ (for $\tilde{b}$ and $\tilde{r} !)$ in $\left[-T, \infty\left[\right.\right.$, which satisfies $\varphi(t) \leqq \frac{8 \hat{c}_{1}}{\hat{c}_{2}} \bar{c}_{5} R \equiv R_{1}$, and we apply the argument described before to this solution [we can suppose of course that $R$ is so small that $R_{1} \leqq \min \left(1 / 2 \hat{c}_{1}, \hat{c}_{3}\right)$, and moreover that $R_{1}$ satisfies (4.28) and (4.36)].

To verify (ii), one has only to look at the compatibility conditions. By a compactness argument one knows that $\left(v^{*}(n T), \sigma^{*}(n T), \omega^{*}(n T)\right)$ converges to $\left(v_{\infty}\right.$, $\left.\sigma_{\infty}, \omega_{\infty}\right)$ in a strong topology; hence, it is easily seen that

$$
\begin{aligned}
& v_{t}^{*}\left(n_{k} T\right) \stackrel{H_{1}(\Omega)}{\longrightarrow} \frac{1}{\sigma_{\infty}+\varrho}\left\{-\nabla\left[p\left(\sigma_{\infty}+\bar{\varrho}, \omega_{\infty}+\bar{\theta}(0)\right)\right]-A v_{\infty}-A \bar{u}(0)\right\} \\
& -\bar{u}_{t}(0)-\left(v_{\infty}+\bar{u}(0)\right) \cdot \nabla\left(v_{\infty}+\bar{u}(0)\right)+b(0) \equiv\left(\dot{v}_{\infty}\right)_{0},
\end{aligned}
$$

and analogously for $\omega_{t}^{*}\left(n_{k} T\right)$. Consequently $\left(\dot{v}_{\infty}\right)_{0} \in H_{0}^{1}(\Omega),\left(\dot{\omega}_{\infty}\right)_{0} \in H_{0}^{1}(\Omega)$ and (ii) is satisfied.

One can prove also that there exists a stationary solution. In fact, by assuming that $b, r, \bar{u}$, and $\bar{\theta}$ do not depend to $t$, we know now that for each $T>0$ there exists a solution which is $T$-periodic. By the stability result, the periodic solutions corresponding to a rational period are always the same, hence there exists a solution which is constant for $t \in \mathbb{Q}$. By a continuity argument this solution does not depend on $t \in \mathbb{R}$ (see [31, Sects. 6 and 7] for similar results for the barotropic case). We have obtained in this way

Theorem 5.1. Let the assumptions 1-5 of Theorem 3.10 be satisfied, and $\bar{u} \cdot \bar{n}_{\mid \Sigma_{\infty}}=0$. Assume also that (4.2) is satisfied for $\gamma$ so small that (4.28) and (4.36) hold. If $b, r, \bar{u}$, and $\bar{\theta}$ are periodic of period $T>0$, then there exists a T-periodic solution $(u, \varrho, \theta)$ of 
$(1.1)_{1}-(1.1)_{3},(1.1)_{5},(1.1)_{7}$ such that $\int_{\Omega} \varrho=\bar{\varrho} \operatorname{vol}(\Omega)$. If $b, r, \bar{u}$, and $\bar{\theta}$ are independent of $t$, then there exists a stationary solution $(u, \varrho, \theta)$ of $(1.1)_{1}-(1.1)_{3},(1.1)_{5},(1.1)_{7}$ such that $\int_{\Omega} \varrho=\bar{\varrho} \operatorname{vol}(\Omega)$. Moreover, these solutions are asymptotically stable and unique among all other solutions $(\mathbf{u}, \mathbf{\varrho}, \boldsymbol{\theta})$ which satisfy (4.37) for $N_{1}$ small enough, and such that $\int_{\Omega} \mathbf{Q}=\bar{\varrho} \operatorname{vol}(\Omega)$.

Remark 5.2. One can also obtain some results about almost-periodicity by following the methods utilized in [14] for the barotropic case.

\section{Appendix}

In this section we want to remark that all the results proved in this paper hold also when the coefficients $\mu, v$, and $\chi$ are not constant, but depend in a suitable way on $\varrho$ and $\theta$. More precisely, we suppose that $\mu \in C^{2}, v \in C^{2}, \chi \in C^{2}$ and that $\mu>0, v \geqq \frac{1}{3} \mu$ and $\chi>0$ for $\varrho>0$ and $\theta>0$.

The proofs of the theorems require some modifications that we want to underline. First of all, the second order operators in $(1.1)_{1}$ and $(1.1)_{3}$ are now

$$
-A u \equiv D_{i}\left(\mu D_{i} u+\mu \nabla u^{i}\right)+\nabla[(v-\mu) \operatorname{div} u]
$$

and

$$
\operatorname{div}(\chi \nabla \theta)
$$

Hence in Sect. 2 we are led to consider the operators

$$
A_{0} v \equiv-D_{i}\left(\mu_{0} D_{i} v+\mu_{0} \nabla v^{i}\right)-\nabla\left[\left(v_{0}-\mu_{0}\right) \operatorname{div} v\right],
$$

and

$$
-\operatorname{div}\left(\chi_{0} \nabla \omega\right)
$$

where $\mu_{0} \equiv \mu\left(\varrho_{0}, \theta_{0}\right), v_{0} \equiv v\left(\varrho_{0}, \theta_{0}\right), \chi_{0} \equiv \chi\left(\varrho_{0}, \theta_{0}\right)$.

The bilinear form associated to $A_{0}$ is no more coercive in $H_{0}^{1}(\Omega)$, but it is easily verified that

$$
\left|\left(A_{0} v, v\right)_{L^{2}}+\lambda(v, v)_{L^{2}}\right| \geqq \frac{\bar{\mu}_{1}}{4}\|D v\|_{0}^{2}
$$

for each $\lambda \in \mathbb{C}$ with $\operatorname{Re} \lambda \geqq\left(c_{*} / \bar{\mu}_{1}^{3}\right)\left\|\nabla \mu_{0}\right\|_{L^{6}(\Omega)}^{4} \equiv \lambda_{0}$. (Here $\left.\bar{\mu}_{1} \equiv \min _{\bar{\Omega}} \mu_{0}, c_{*}=c_{*}(\Omega)\right)$. Remark that $\varrho_{0} \in H^{2}(\Omega), \theta_{0} \in H^{5 / 2}(\Omega), \quad 0<m \leqq \varrho_{0}(x) \leqq M, \quad 0<\theta_{3} \leqq \theta_{0}(x) \leqq \theta_{4}$, hence $\lambda_{0}<+\infty$ and $\bar{\mu}_{1}>0$.

Moreover, following the usual regularization procedure, one gets the a priori estimates,

$$
\|v\|_{k+2}^{2} \leqq c\left(1+\left\|D \mu_{0}\right\|_{1}^{a}+\left\|D v_{0}\right\|_{1}^{a}\right)\left(\left\|A_{0} v\right\|_{k}^{2}+\|v\|_{0}^{2}\right), \quad k=0,1,
$$

where $a$ is a suitable positive integer, and $c$ depends on $\Omega$ and in an increasing way on $1 / \bar{\mu}_{1}, \bar{\mu}_{2} \equiv \max _{\bar{\Omega}} \mu_{0}$ and $\bar{v}_{2} \equiv \max _{\bar{\Omega}} v_{0}$. An analogous result holds also for $\omega$.

The proof of Lemmas 2.1 and 2.3 must be changed somewhere, since now $\|v\|_{0}^{2}$ appears on the right-hand side of (6.5), and $D_{i}\left(\mu_{0} \nabla v^{i}\right)$ gives some additional terms 
when it is integrated by parts. Moreover, on the right-hand side of $(2.1)_{1}$ we have now also

$$
A_{0} \tilde{v}-\left\{-D_{i}\left(\tilde{\mu} D_{i} \tilde{v}+\tilde{\mu} \nabla \tilde{v}^{i}\right)-\nabla[(\tilde{v}-\tilde{\mu}) \operatorname{div} \tilde{v}]\right\},
$$

where $\tilde{\mu} \equiv \mu(\tilde{\sigma}+\bar{\varrho}, \tilde{\omega}+\bar{\theta}), \tilde{v} \equiv v(\tilde{\sigma}+\varrho \bar{\varrho}, \tilde{\omega}+\bar{\theta})$ [and something of this kind on the right-hand side of $(2.3)_{1}$, too].

However, having taken into account these modifications, the local existence theorem still holds under the same assumptions.

With regard to the global a priori estimates and to the asymptotic stability, we have only to remark that in $(3.1)_{1},(3.3)_{1},(4.6)_{1}$, and $(4.8)_{1}$ we set now

$$
\bar{A} \equiv-\frac{\mu\left(\bar{\varrho}, \theta_{1}\right)}{\bar{\varrho}} \Delta-\frac{v\left(\bar{\varrho}, \theta_{1}\right)}{\bar{\varrho}} \nabla \operatorname{div},
$$

and

$$
\bar{\chi} \equiv \frac{\chi\left(\bar{\varrho}, \theta_{1}\right)}{\varrho c_{v}\left(\varrho, \theta_{1}\right)} .
$$

The additional expressions which appear on the right-hand side of $(3.1)_{1},(3.3)_{1}$, $(4.6)_{1}$, and $(4.8)_{1}$ can be estimated in the same way as in the previous situation, and consequently all the results exposed in this paper are still valid.

\section{References}

1. Agmon, S., Douglis, A., Nirenberg, L.: Estimates near the boundary for solutions of elliptic partial differential equations satisfying general boundary conditions. II. Commun. Pure Appl. Math. 17, 35-92 (1964)

2. Beirão da Veiga, H.: Diffusion on viscous fluids. Existence and asymptotic properties of solutions. Ann. Sc. Norm. Super. Pisa, Cl. Sci.(IV) 10, 341-355 (1983)

3. Cattabriga, L.: Su un problema al contorno relativo al sistema di equazioni di Stokes. Rend. Semin. Mat. Univ. Padova 31, 308-340 (1961)

4. Fiszdon, W., Zajaczkowski, W.M.: The initial boundary value problem for the flow of a barotropic viscous fluid, global in time. Appl. Anal. 15, 91-114 (1983)

5. Fiszdon, W., Zajaczkowski, W.M.: Existence and uniqueness of solutions of the initial boundary value problem for the flow of a barotropic viscous fluid, local in time. Arch. Mech. 35, 497-516 (1983)

6. Fiszdon, W., Zajaczkowski, W.M.: Existence and uniqueness of solutions of the initial boundary value problem for the flow of a barotropic viscous fluid, global in time. Arch. Mech. 35, 517-532 (1983)

7. Giaquinta, M., Modica, G.: Nonlinear systems of the type of the stationary Navier-Stokes system. J. Reine Angew. Math. 330, 173-214 (1982)

8. Graffi, D.: II teorema di unicità nella dinamica dei fluidi compressibili. J. Rat. Mech. Anal. 2, 99-106 (1953)

9. Itaya, N.: On the Cauchy problem for the system of fundamental equations describing the movement of compressible viscous fluids. Kodai Math. Sem. Rep. 23, 60-120 (1971)

10. Judovič, V.I.: A two-dimensional problem of unsteady flow of an ideal incompressible fluid across a given domain. Am. Math. Soc. Trans. (2) 57, 277-304 (1966) [previously in Mat. Sb. (N.S.) 64(106), 562-588 (1964) (in Russian)]

11. Lions, J.L., Magenes, E.: Problèmes aux limites non homogènes et applications, 1. Paris: Dunod 1968 
12. Lukaszewicz, G.: An existence theorem for compressible viscous and heat conducting fluids. Math. Meth. Appl. Sci. 6, 234-247 (1984)

13. Lukaszewicz, G.: On the first initial-boundary value problem for the equations of motion of viscous and heat conducting gas. Arch. Mech. 36(1984) (to appear)

14. Marcati, P., Valli, A.: Almost-periodic solutions to the Navier-Stokes equations for compressible fluids. Boll. Unione Mat. Ital. (to appear)

15. Matsumura, A., Nishida, T.: The initial value problem for the equations of motion of viscous and heat-conductive gases. J. Math. Kyoto Univ. 20, 67-104 (1980)

16. Matsumura, A., Nishida, T.: The initial value problem for the equations of motion of compressible viscous and heat-conductive fluids. Proc. Jpn. Acad. Ser. A 55, 337-342 (1979)

17. Matsumura, A., Nishida, T.: The initial boundary value problem for the equations of motion of compressible viscous and heat-conductive fluid. Preprint University of Wisconsin, MRC Technical Summary Report no. 2237 (1981)

18. Matsumura, A., Nishida, T.: Initial boundary value problems for the equations of motion of general fluids. In: Computing methods in applied sciences and engineering, V, Glowinski, R., Lions, J.L. (ed.). Amsterdam, New York, Oxford: North-Holland 1982

19. Matsumura, A., Nishida, T.: Initial boundary value problems for the equations of motion of compressible viscous and heat-conductive fluids. Commun. Math. Phys. 89, 445-464 (1983)

20. Nash, J.: Le problème de Cauchy pour les équations différentielles d'un fluide général. Bull. Soc. Math. Fr. 90, 487-497 (1962)

21. Padula, M.: Existence and uniqueness for viscous steady compressible motions. Arch. Ration. Mech. Anal. (to appear)

22. Secchi, P., Valli, A.: A free boundary problem for compressible viscous fluids. J. Reine Angew. Math. 341, 1-31 (1983)

23. Serrin, J.: Mathematical principles of classical fluid mechanics, in "Handbuch der Physik", Bd. VIII/1. Berlin, Göttingen, Heidelberg: Springer 1959

24. Serrin, J.: On the stability of viscous fluid motions. Arch. Ration. Mech. Anal. 3, 1-13 (1959)

25. Serrin, J.: A note on the existence of periodic solutions of the Navier-Stokes equations. Arch. Ration. Mech. Anal. 3, 120-122 (1959)

26. Serrin, J.: On the uniqueness of compressible fluid motions. Arch. Ration. Mech. Anal. 3, 271-288 (1959)

27. Solonnikov, V.A.: Solvability of the initial-boundary value problem for the equations of a viscous compressible fluid. J. Sov. Math. 14, 1120-1133 (1980) [previously in Zap. Nauchn. Semin. Leningr. Otd. Mat. Inst. Steklova (LOMI) 56, 128-142 (1976) (in Russian)]

28. Tani, A.: On the first initial-boundary value problem of compressible viscous fluid motion. Publ. Res. Inst. Math. Sci. 13, 193-253 (1977)

29. Valli, A.: Uniqueness theorems for compressible viscous fluids, especially when the Stokes relation holds. Boll. Unione Mat. Ital., Anal. Funz. Appl. (V) 18-C, 317-325 (1981)

30. Valli, A.: An existence theorem for compressible viscous fluids. Ann. Mat. Pura Appl.(IV) 130, 197-213 (1982); (IV) 132, 399-400 (1982)

31. Valli, A.: Periodic and stationary solutions for compressible Navier-Stokes equations via a stability method. Ann. Sc. Norm. Super. Pisa (IV) 10, 607-647 (1983)

32. Vol'pert, A.I., Hudjaev, S.I.: On the Cauchy problem for composite systems of nonlinear differential equations. Math. USSR-Sb 16 517-544 (1972) [previously in Mat. Sb. (N.S.) 87 (129), 504-528 (1972) (in Russian)]

Communicated by L. Nirenberg

Received April 24, 1985; in revised form July 15, 1985 